\title{
MicroRNAs and the PTEN/PI3K/Akt pathway in gastric cancer (Review)
}

\author{
MINGLI HU, SHIXUAN ZHU, SHENGWEI XIONG, XINGXING XUE and XIAODONG ZHOU \\ Department of Gastroenterology, First Affiliated Hospital of Nanchang University, \\ Nanchang, Jiangxi 330006, P.R. China
}

Received June 30, 2018; Accepted November 28, 2018

DOI: $10.3892 / o r .2019 .6962$

\begin{abstract}
Gastric carcinogenesis arises from complicated interactions among host, environmental and bacterial factors, which cause genetic and epigenetic dysregulation of oncogenic and tumor-suppressive genes. MicroRNAs (miRNAs), a class of small non-coding RNAs that post-transcriptionally regulate $\sim 30 \%$ human genes, may serve as oncogenes or tumor-suppressors in malignancies, including gastric cancer (GC). Although miRNA dysregulation commonly exists in GC, exact roles miRNAs serve in the pathogenesis and promotion of this tumor remain undetermined. Recently, results of previous studies regarding mechanisms underlying miRNAs generally converged on pathways critical in cellular processes, including cell proliferation, apoptosis and invasion, among which phosphatase and tensin homolog (PTEN)/phosphatidylinositol 3-kinase (PI3K)/protein kinase B (Akt) signaling is a fundamental one, with frequent oncogenic alterations in GC. Therefore, in the present review, the disorder and function of miRNAs and PTEN/PI3K/Akt signaling in GC are discussed. Additionally, how miRNAs transduce their effects by regulating this pathway, particularly in GC stem cells and the tumor microenvironment, and two novel hypotheses significant in carcinogenesis, tumor progression and recurrence, are discussed. Furthermore, the roles of miRNAs and the PTEN/PI3K/Akt pathway in target therapies against this lethal disease are outlined.
\end{abstract}

\section{Contents}

1. Introduction

2. miRNAs in GC

Correspondence to: Dr Xiaodong Zhou, Department of Gastroenterology, First Affiliated Hospital of Nanchang University, 17 Yong Waizheng Street, Nanchang, Jiangxi 330006, P.R. China E-mail: yfyzxd@163.com

Key words: gastric cancer, microRNAs, the phosphatase and tensin homolog/phosphatidylinositol 3-kinase/protein kinase B pathway, mechanism, clinical application
3. PTEN/PI3K/Akt pathway in GC

4. Regulation of miRNAs on the PTEN/PI3K/Akt pathway in GC

5. Conclusion

\section{Introduction}

Gastric cancer (GC) is the fourth most common cancer and the second leading cause of cancer-associated mortality worldwide (1). The incidence and mortality rates of GC are the highest in East Asia, primarily in China (1-3). Gastric carcinogenesis is a complex process, which involves crosstalk among host, environmental and bacterial factors, leading to different molecular alterations at the genetic and epigenetic level; Helicobacter pylori is a well-recognized high-risk factor $(4,5)$. Gastrectomy is the primary strategy for patients with early-stage GC. However, the absence of specific symptoms in the early-stage leads to the majority of patients with GC diagnosed in the unresectable stage, and systemic chemotherapy is the primary option for these patients (6). Chemotherapy resistance frequently emerges as a cause of treatment failure (6). At present, the outcome for patients with advanced GC remains poor, with 5-20\% 5-year survival and a median overall survival of 10 months (3). Therefore, examining novel biomarkers for early diagnosis and other feasible treatments based on a better understanding of mechanisms underlying GC pathogenesis, in addition to chemoresistance, is urgently required.

MicroRNAs (miRNAs) represent a large group of conserved small non-coding RNAs (ncRNAs) with a length of 17-25 nucleotides, which bind to the 3'-untranslated region (UTR) of mRNAs of their target genes, silencing expression by cleaving the mRNA molecules or inhibiting their translation $(1,5)$. In this manner, $\sim 30 \%$ human genes are modulated by miRNAs; the majority are directly or indirectly implicated in signaling pathways fundamental in cellular activities, including proliferation, differentiation, apoptosis and migration $(7,8)$. As a result, miRNAs are crucial regulators in the initiation and progression of various diseases, particularly cancer. In GC, dysregulated oncogenic or tumor-suppressive miRNAs promote malignant phenotypes, including tumor growth, metastasis, angiogenesis and drug-resistance, by regulating downstream targets and associated pathways $(4,9,10)$. 
The phosphatase and tensin homolog (PTEN)/phosphatidylinositol 3-kinase (PI3K)/protein kinase B (Akt) signaling pathway represents a crucial one $(11,12)$. PTEN, a dual protein and lipid phosphatase, primarily dephosphorylates phosphatidylinositol-3,4,5-trisphosphate (PIP3), which is the product of PI3K and is able to recruit Akt to the membrane, where it is phosphorylated and stimulated by other kinases dependent on PIP3 (13). Activated Akt may regulate multiple biological processes, including cell survival, metabolism, cell proliferation and growth, by affecting its downstream substrates (13-15). Genetic and epigenetic alterations occurring in a number of components of this pathway lead to its constitutive activation in human cancer, including GC $(11,16)$. Furthermore, the PTEN/PI3K/Akt pathway may be regulated by miRNAs in GC, suggesting that this signaling serves an essential role in mediating oncogenic effects of dysregulated miRNAs during the onset and development of this disease $(6,8)$. In the present review, the dysregulation and function of miRNAs, in addition to genetic alterations and the roles of the PTEN/PI3K/Akt pathway in GC are summarized. Furthermore, how this signaling serves as an important mediator of miRNAs is discussed. Based on their involvement in the mechanism underlying gastric carcinogenesis and progression, the clinical applications of miRNAs and its signaling as biomarkers or therapeutic targets in GC management are additionally discussed.

\section{2. miRNAs in GC}

Dysregulation and function of miRNAs in GC. Accumulating evidence has documented the overexpression or downregulation of specific miRNAs in GC. Due to the extensive regulatory function of miRNAs in gene expression, the dysregulated miRNAs may result in oncogenic activities regarding approximately all aspects of tumorigenesis and progression, including cell proliferation, apoptosis, invasion and migration $(1,4)$. Generally, oncogenic miRNAs contribute to tumor development with their aberrantly high expression in GC, whereas, the silenced or lost expression of tumor-suppressive miRNAs may additionally exert positive effects during oncogenesis $(1,4)$. Carcinogenic effects of miRNAs have been acknowledged to be the result of their disordered post-transcriptional regulation of oncogenic and tumor suppressive genes via complementary base-pairing $(1,4)$. The aberrant expression level of miRNAs, implicated oncogenic effects and associated target genes in GC are summarized in Table I.

Tsukamoto et al (17) detected miRNA expression in 22 surgically resected GC tissues and identified that 39 miRNAs exhibited different expression levels between tumor and normal tissues, among which six miRNAs were downregulated; whereas, the other 33 miRNAs were upregulated in GC. miR-28 was demonstrated to be upregulated in $31 \mathrm{GC}$ tissues compared with the matched adjacent non-tumor tissues $(\mathrm{P}<0.05)$, and the higher expression of miR-28 has been additionally observed in a series of GC cell lines compared with the normal control (5). miR-28 contributed to GC cell proliferation and invasion by targeting and downregulating tumor suppressor PTEN (5). Caudal-related homeobox 1 (CDX1), an intestinal-specific transcription factor important in gastric intestinal metaplasia, could significantly repress GC cell growth by inducing cell cycle arrest and apoptosis (10). CDX1 was targeted by oncogenic miR-296-5p, which was detected to be overexpressed in GC tissues and abolished the suppressive effects induced by CDX1 (10). Different from the elevated expression level of oncogenic miRNAs in GC, the expressions of tumor-suppressor miRNAs, including miR-137, miR-34a, miR-15a and miR-16-1, which exert negative regulations on cell proliferation, epithelial-mesenchymal transition (EMT), migration, invasion, colony formation in vitro, in addition to tumorigenicity and metastasis in vivo, have been documented to be evidently decreased in the tumor tissues and cell lines compared with normal controls (18-21). The targets responsible for their effects including Akt2, platelet-derived growth factor receptor (PDGFR) and twist family bHLH transcription factor 1 (18-21). The synergistic regulation of multiple genes, including phosphoinositide-3-kinase regulatory subunit 2 (PI3KR2), CRK proto-oncogene, adaptor protein, vascular cellular adhesion protein 1 and serine/threonine-protein kinase PLK2, affects the miR-126 diverse antitumor effect of restraining tumor cell growth, migration and invasion, and inducing cell cycle arrest in $\mathrm{G}_{0} / \mathrm{G}_{1}$ phase, apoptosis in vitro, in addition to inhibiting tumorigenicity, angiogenesis and metastasis in vivo; however, during gastric oncogenesis, the expression of miR-126 was significantly decreased (22-24). Otsubo et al (25) observed that miR-126 was aberrantly upregulated in a number of GC cell lines and tumor tissues, and miR-126 overexpression contributed to growth and colony formation of GC cells by modulating its tumor-suppressive target sex-determining region Y-box 2.

\section{Possible clinical application of miRNAs in GC}

miRNAs as promising biomarkers for GC. As mentioned, the significantly different expression of miRNAs may be detected between tumor and normal gastric mucosa tissues. When detecting samples from patients with GC with different clinicopathological characteristics and prognosis, the expression level of specific miRNAs may additionally be distinct. The expression of miR-10b and miR-21 was markedly higher in lymphoma node metastasis-positive tumor tissues compared with lymphoma node metastasis-free tumor tissues $(26,27)$. The low expression of tumor suppressor miR-1 and miR-146a was associated with vascular invasion, lymph node involvement and distant metastasis, in addition to the poor prognosis of patients with GC. Furthermore, the relative level of miR-146a expression was an independent predictive factor for overall survival $(28,29)$. Therefore, miRNA expression levels in tumor tissues may be used to diagnose patients with GC, in addition to distinguishing patients with different prognosis and developing treatment strategies.

Additionally, circulating miRNAs, which were contained in secretive exosomes, may be detected in body fluids, including sera, plasma, urin and gastric juice (1). Due to their characteristics, including high stability, close association with disease statuses and ease of measurement, circulating miRNAs have been investigated in multiple diseases, particularly cancer, regarding their promising functionality as novel non-invasive biomarkers (1). Liu et al (30) reported that serum exosome miR-451 of patients with GC was significantly increased compared with healthy controls. Furthermore, the high expression level of exosome miR-451 was associated 


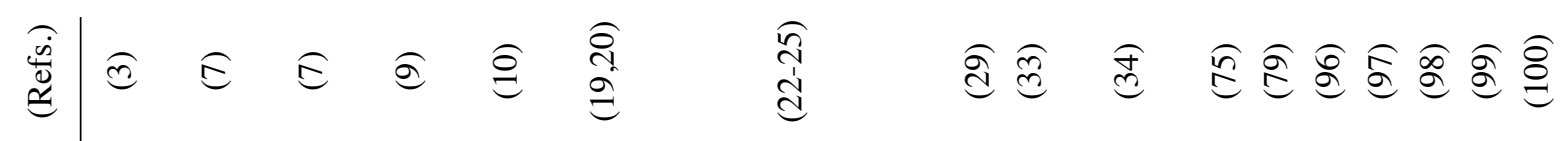

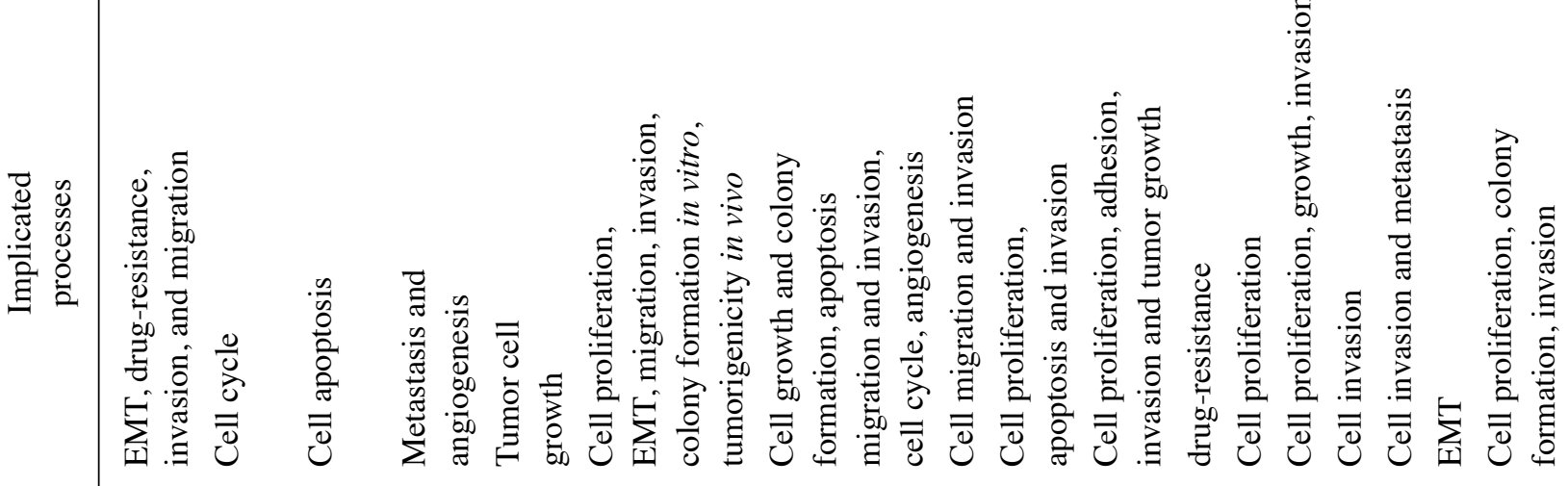

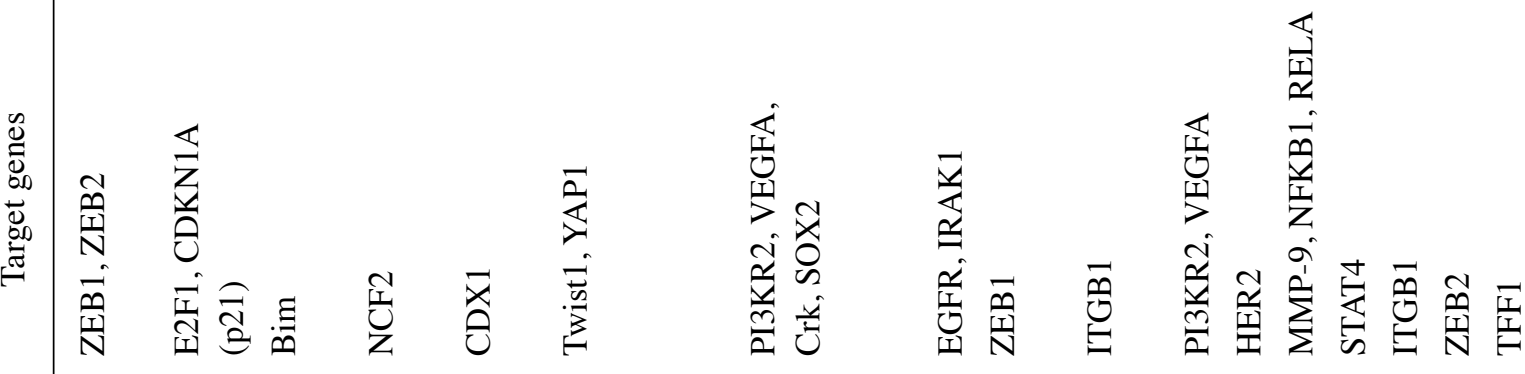

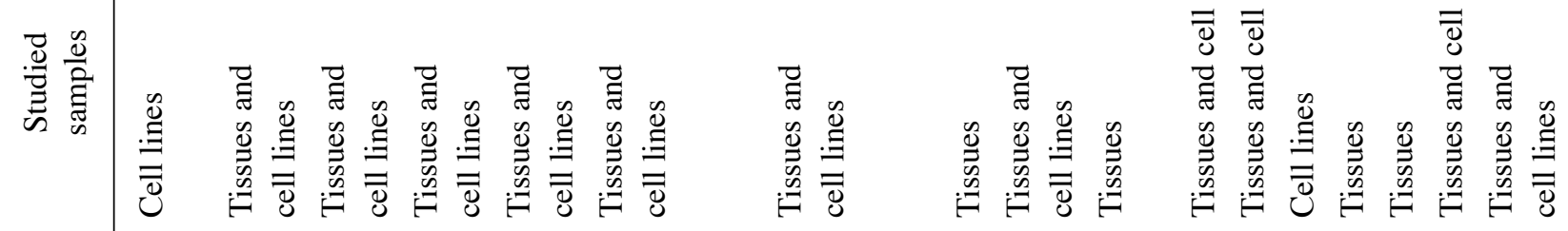

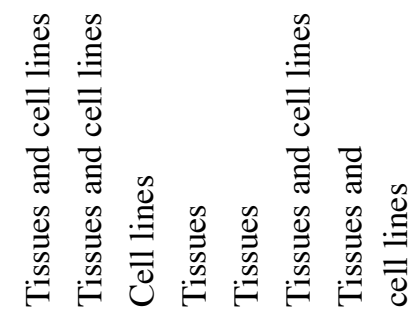

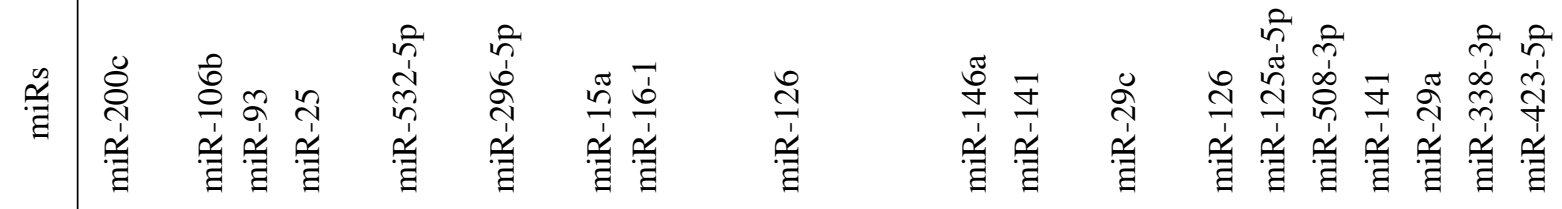

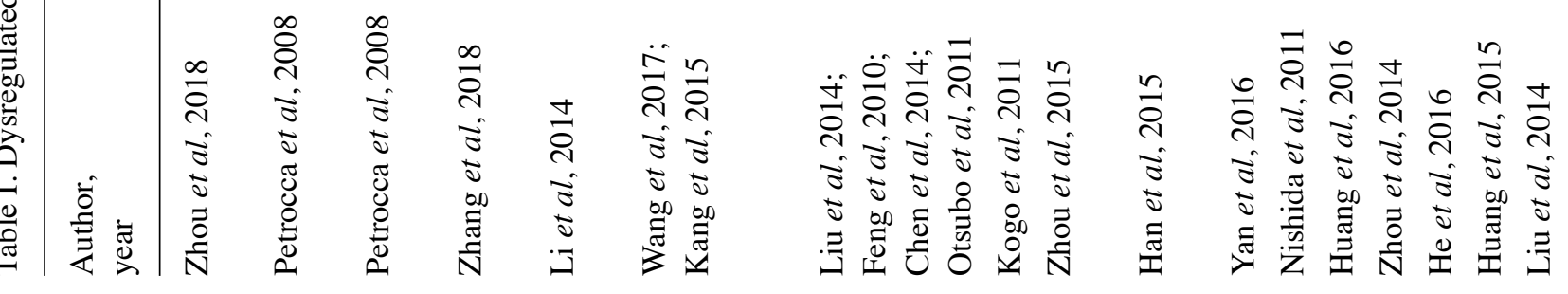


with poor differentiation, in addition to high proliferation and metastasis potential of GC, and may further predict poor outcomes of patients with GC post-surgery (30). Zhu et al (31) identified an miRNA signature with the capacity to diagnose early-stage GC accurately, which consisted of five miRNAs (miR-16, miR-25, miR-92a, miR-451 and miR-486-5p) overexpressed in the plasma of patients with GC.

miRNAs as potential therapeutic targets for GC. As the dysregulated miRNAs may contribute to the pathogenesis of $\mathrm{GC}$, restoring the level of downregulated tumor-suppressive miRNAs and/or upregulated oncogenic miRNAs, they may represent a potential treatment strategy against the tumor. Consistently, when transfecting GC cells with an miR-590-5p inhibitor, significantly decreased proliferative and invasive abilities, in addition to increased sensitivity to cisplatin (DDP) and paclitaxel (PTX) were observed (32). Whereas, ectopic expression of miR-141 in tumor cells may lead to $40 \%$ inhibition of proliferation and prominent reduction in invasion (33). In addition to the in vitro studies, miRNAs have been tested for their treatment efficacy in vivo. Han et al (34) identified that in a xenograft nude mouse model, tumor growth in mice implanted with miR-29c overexpressing GC cells was significantly slower compared with mice treated with untreated parental cells $(\mathrm{P}<0.0001)$, and injecting miR-29c mimics intratumorally additionally resulted in a marked inhibition of tumor growth. According to the results of the bioluminescence imaging and analysis, and the number of lung metastasis in mice, miR-137 was demonstrated to exert anti-metastatic effects in vivo (21).

\section{PTEN/PI3K/Akt pathway in GC}

Components of the PTEN/PI3K/Akt pathway. PTEN is a dual lipid and protein phosphatase and a common tumor suppressor. Since its identification, the mutational and epigenetic silencing of PTEN have been documented in various cancer types, owing to its extensive function in critical cellular processes, which may be generally divided into two categories, cell growth/survival and cell migration/adhesion, regulated through its lipid and protein phosphatase activities, respectively $(12,13)$. The primary substrate of PTEN is PIP3, the product of PI3Ks, a lipid kinase family with the typical ability to phosphorylate the 3'-OH group in inositol phospholipids on the cell membrane (35). PI3Ks generally consist of three classes (class I, class II and class III) (35). Class I PI3Ks, heterodimeric proteins comprising a catalytic subunit and a regulator subunit, may be further subdivided into subclass IA and subclass IB, which may be activated by tyrosine kinase receptors (RTKs) and guanosine-binding protein coupled receptors (GPCR), respectively (11). Among the three classes, only class I PI3Ks were identified to be implicated in human cancer (35) and is the one primarily referred to in the present review.

Akt, additionally termed $\mathrm{PKB}$, is a serine-threonine kinase downstream of PTEN/PI3K signaling. Akt includes three isoforms transcribed from different genes, $\mathrm{Akt} 1 / \mathrm{PKB} \alpha$, Akt $2 / \mathrm{PKB} \beta$ and Akt $3 / \mathrm{PKB} \gamma($ (14). Each member comprises three conserved domains; a plekstrin homology $(\mathrm{PH})$ domain in the $\mathrm{N}$-terminal, a central kinase domain and a hydrophobic 
C-terminal tail (14). In the first and the third domain, a regulatory site exists, which is necessary for the PI3K-dependent activation of Akt (Thr308 and Ser473 in Akt1) (36).

Activation process of the PTEN/PI3K/Akt pathway. The stimulation of GPCRs or RTKs [including IGFR, PDGFR, epidermal growth factor receptor (EGFR) and c-Met] by various stimuli, including growth factors, hormones and extracellular matrix (ECM) components, initiates the activation of the PI3K/Akt pathway (14). The interaction of the Src homology 2 (SH2) domains in the regulatory subunit of PI3K with the intracellular section of the activated cell-surface receptors or their adaptor proteins leads to the allosteric activation of the PI3K catalytic subunit $(37,38)$. Alternatively, the activated Ras may stimulate PI3K by binding to a Ras binding domain (RBD) located at p110 catalytic subunits (39). Subsequently, activated PI3K associates and phosphorylates lipids, which in turn phosphorylates phosphatidylinositol-4,5-bisphosphate (PIP2) at the inner side of the plasma membrane into phosphatidylinositol-3,4,5-trisphosphate (PIP3), a significant second messenger in cells (14). The production of PIP3 results in the recruitment of proteins containing a $\mathrm{PH}$ domain to cellular membranes, including Akt and its activating kinase 3-phosphoinositide-dependent protein kinase 1 (PDK1), which are critical for transducing the activity of PI3K (40). It is generally acknowledged that the conformational alterations of Akt, which refers to the exposure of its two primary regulatory residues, may occur following the interaction of the PH domain with PIP3. The PH domain is additionally accountable for the heterodimerization of $\mathrm{Akt}$ and PDK1, which results in activation of Akt through the phosphorylation of PDK1 at Thr308 of Akt (14). However, apart from the phosphorylation of this residue, full activation of $\mathrm{Akt}$ still requires phosphorylation of the other regulatory site in the carboxyl-terminal hydrophobic motif, Ser473, which is fulfilled by mammalian target of rapamycin (mTOR) complex 2 , additionally termed PDK2 $(11,41)$. Subsequently, activated Akt will be translocated into the nucleus to phosphorylate its downstream substrates involved in multiple cellular processes, including apoptosis, cell cycle progression, metastasis and metabolism $(14,42)$.

Among multiple substrates, PTEN primarily targets and dephosphorylates lipid PIP3 at the D3 position of the inositol ring, and thus serves as the primary negative regulator of PI3K/AKT signaling by reducing PIP3 production and inhibiting subsequent recruitment along with activation of Akt (14). In the same manner, other phosphatases may additionally block the activation of PI3K/Akt signaling, including SH2-containing inositol 5-phosphatase and inositol polyphosphate 4-phosphatase type II (37). Carboxyl-terminal modulator protein, a protein partner which binds to the $\mathrm{C}$ terminus of Aktl at the plasma membrane, is able to block the phosphorylation on Ser473 and Thr308, thus reducing the activation of Akt (43).

Genetic alterations of the PTEN/PI3K/Akt pathway in GC. Genetic alterations of different codes of PTEN/PI3K/Akt signaling may be frequently detected in GC (Table II), which contribute to the overactivation of this signaling (11). However, as genetic analysis is a reliable experiment strategy for validating specific genes involved in pathologic processes, these documented genetic alterations may reflect the involvement of the PTEN/PI3K/Akt pathway in gastric carcinogenesis and progression.

Loss of the tumor suppressor PTEN, was considered a common mechanism for the activation of Akt signaling and inversely, the constitutive activation of Akt was demonstrated to be largely responsible for PTEN-mediated carcinogenesis $(14,44)$. The mutational inactivation of PTEN may be identified in numerous carcinomas, including GC (45). Wen et al (46) identified mutations of PTEN in 27 of 144 patients with GC, and the mutation rate was higher in advanced tumor, node and metastasis (TNM) stages in addition to poorly differentiated ones, which may account for the downregulated PTEN expression and the activation of PI3K/Akt signaling detected in tumor tissues. Epigenetically silencing PTEN by mesylating 5' CpG islands in the promoter possibly accounts for the its downregulated expression in GC (16). Phosphatidylinositol-4,5-bisphosphate 3-kinase catalytic subunit $\alpha$ (PIK3CA), the gene encoding PI3K catalytic subunit $\mathrm{p} 110 \alpha$, has been documented to be one of the most frequently mutated genes in human cancer (42). Samuels et al (47) identified activating mutations of PIK3CA in numerous cancer types, among which the mutation rate in GC was $25 \%$ ( 3 in 12 cases), and $>75 \%$ of the mutations occurred in two small clusters located in the helical and kinase domains, causing the significant upregulation of lipid kinase activity and the resultant stimulation of downstream Akt signaling. In addition to mutations, the genomic amplification of PIK3CA was additionally an important mechanism underlying the oncogenic activation of the PI3K/Akt pathway in malignant diseases (14). Shi et al (48) determined that PIK3CA amplification in 88 of 131 tested samples (67\%) was closely correlated with the elevated activation of the PI3K pathway, in addition to a poor outcome for patients with GC. Furthermore, in the same previous study, the PIK3CA amplification rate was significantly higher compared with the mutation rate $[8 / 113$ (7.1\%)] (48). The genetic alteration, including mutation and amplification, may additionally occur to Akt in GC, although the documented incidence is low.

Function of the PTEN/PI3K/Akt pathway in GC. PI3K signaling is vital in regulating multiple fundamental cellular activities, including cell proliferation, apoptosis and metastasis (49). With frequent alterations identified in GC, the PTEN/PI3K/Akt pathway is significantly involved in gastric carcinogenesis and progression $(11,50)$.

The disturbed balance between cell growth and apoptosis leads to tumorigenesis, is a result from the disorder of complex regulation networks, comprising of pivotal proteins and signaling cascades. The PTEN/PI3K/Akt pathway is critical due to its fundamental role in the decision of cell death and survival $(14,42)$. Pro-survival effects of Akt signaling have been identified in previous studies using antitumor factors. Zhao et al (51) identified that overexpression of a tumor suppressor gene, inhibitor of growth 3 , in GC cells was able to reduce cell proliferation and arrest the cell cycle at $\mathrm{G}_{2} / \mathrm{M}$ phase, in addition to induce cell apoptosis, and the impairment of PI3K/Akt signaling was the involved mechanism. Geridonin and paclitaxel was able to serve synergistically to suppress Akt signaling by inhibiting Akt phosphorylation at Thr308 


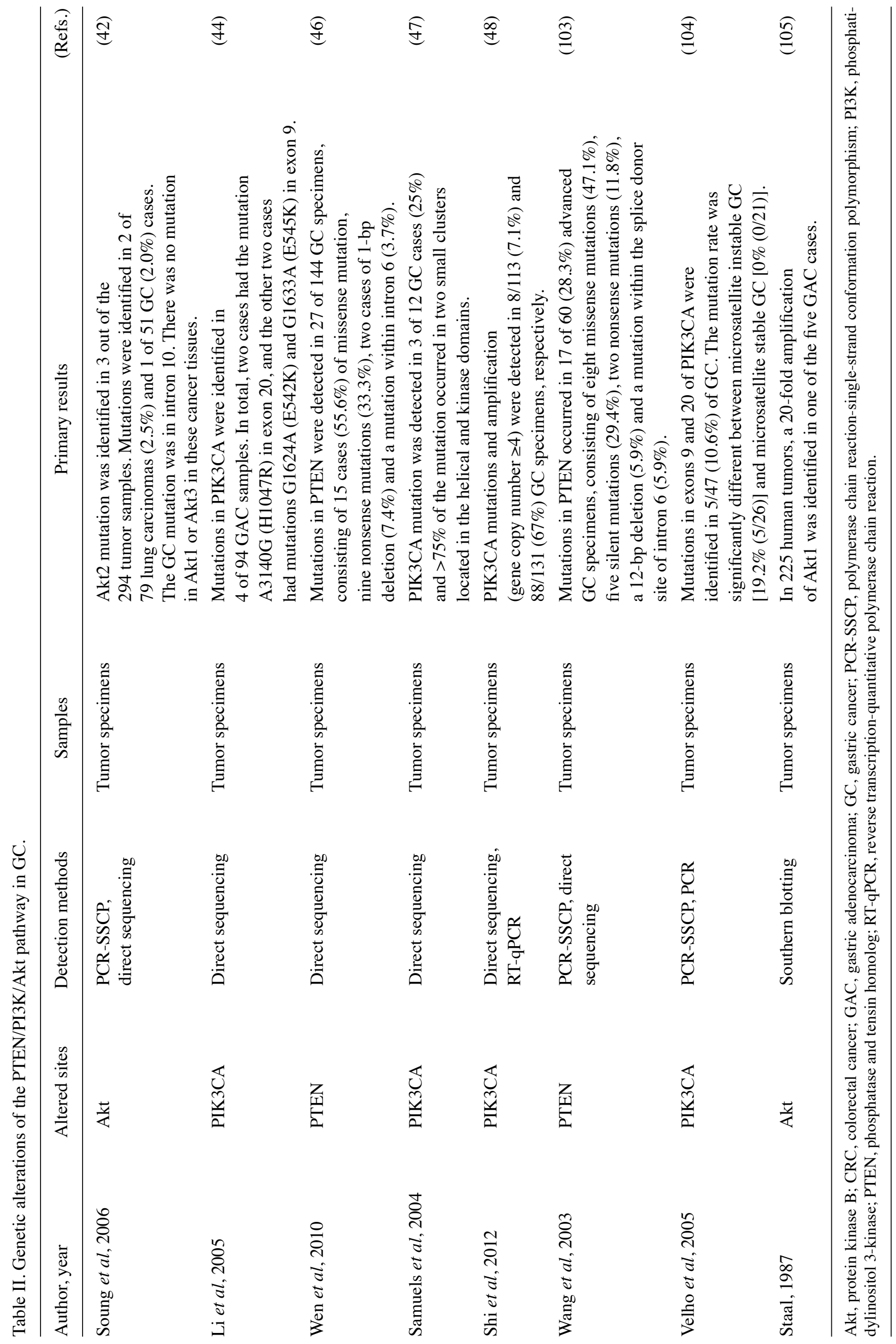


and Ser473, in addition to elevating the PTEN expression level, which led to the accumulation of p53 and pro-apoptotic apoptosis regulator Bcl-2 (Bcl-2) family members, together with downregulation of anti-apoptotic members (52). These molecular alterations accounted for the enhanced apoptosis and repressed growth in vitro and in vivo induced by this combination therapy against GC (52).

Apart from contributing to the initiation of GC, the aberrantly activated PI3K signaling was additionally able to contribute to the progression of GC into highly malignant types characterized by metastasis and resistance to chemotherapy. Metastasis is a high potential risk and the predominant cause of recurrence and mortality among patients with GC, and a number of cell biological activities are controlled in this multistep process, including adhesion, migration, invasion and angiogenesis (1). Lectin-like oxidized low-density lipoprotein receptor-1 was overexpressed in GC cells and increased the migratory and invasive ability by enhancing EMT, and activating the PI3K/Akt/glycogen synthase kinase $3 \beta$ (GSK3 $\beta$ ) pathway (53). Vascular endothelial growth factor-A (VEGF-A) was suggested to promote angiogenesis by activating the downstream Akt/mTOR cascade in GC (24). Phosphatase of regenerating liver-3 (PRL-3), a protein tyrosine phosphatase, silenced PTEN by reducing its expression and enhancing its phosphorylation, leading to the activation of PI3K/Akt signaling and upregulation of matrix metalloproteinase (MMP)-2/MMP-9, through which PRL-3 contributed to the lethal peritoneal metastasis of GC (49). Chen et al (54) documented the enrichment and activation of PI3K/Akt/mTOR signaling along with decreased PTEN expression in GC cells resistant to PTX, a microtubule stabilizer widely used in the front-line chemotherapy for patients with advanced GC. The low response to other antitumor agents, including DDP and 5-fluorouracil (5-FU), may additionally be attributed to the stimulated PI3K signaling $(6,11,32)$.

Anticancer drugs targeting the PTEN/PI3K/Akt pathway. As a most frequently dysregulated pathway in cancer, the PTEN/PI3K/Akt pathway has attracted increasing attention for its potential in target therapies for a number of malignancies. In this context, $>40$ inhibitors against different points of this pathway, primarily PI3Ks, Akt and mTOR, reached various stages of clinical trials; the mTOR inhibitors, temsirolimus and everolimus, in addition to the PI3K inhibitors, idelalisib and copanlisib, have been approved by the Food and Drug Administration for clinical anticancer treatment (38). Consistently, these therapies have additionally been investigated for GC in numerous previous studies regarding treatment effects of inhibiting tumor growth, metastasis and modifying chemo-sensitivity, including LY294002, a commonly used PI3K inhibitor, and the Akt inhibitor MK-2206 (6,49,55). A number of effective and safe inhibitors, including BKM120 targeting PI3K and everolimus against mTOR, were enrolled in the clinical studies of different phases among patients with GC (11) Antibodies or inhibitors against the RTKs or the ligands have additionally been examined as potential target therapies for GC. Trastuzumab, a humanized monoclonal antibody against human epidermal growth factor receptor 2 (HER2), is the first molecular target drug in GC and may be combined with chemotherapy as a standard treatment for cases with HER2 overexpression/amplification, which affect $11-20 \%$ patients with GC (3). Furthermore, it was observed that the combined use of trastuzumab and LY294002 may exhibit a synergistic repression on downstream Akt signaling in GC cells (56). Ramucirumab and apatinib, inhibitors of vascular endothelial growth factor receptor-2 (VEGFR-2), have additionally been approved as anti-angiogenic therapies for advanced GC (57).

\section{Regulation of miRNAs on the PTEN/PI3K/Akt pathway in GC}

miRNAs regulate PI3K/Akt signaling. The activated $\mathrm{PI} 3 \mathrm{~K}$ signaling contributed to the initiation and development of GC through involvement in different cellular activities. Multiple evidence has demonstrated that miRNAs were highly implicated in the regulation of the PI3K/Akt pathway by targeting this pathway (Table III; Fig. 1), which partially identified the mechanisms underlying the oncogene or tumor-suppressor role of miRNAs in GC.

During gastric carcinogenesis, the oncogenic effects caused by the overexpressed oncogenic miRNAs or downregulated tumor-suppressive miRNAs may be attributed to the aberrantly activated PI3K/Akt signaling. It was observed that miR-196b promoted GC tumor growth through its promotion in the cell cycle and cell proliferation, possibly by stimulating the PI3K/Akt/mTOR pathway (58). Conversely, it was identified that the tumor suppressor miR-203, inhibited GC cell proliferation by targeting PIK3CA and consequently attenuated activation of Akt (59). Tsukamoto et al (17) observed that ectopic expression of miR-375 lead to a decrease in PDK1 expression and a subsequent decline in phosphorylation of Akt at Ser473 and Thr308. Furthermore, the dysregulated expression level of an anti-apoptosis substrate of Akt, E3 ubiquitin-protein ligase XIAP, along with the increased activity of specific apoptosis executioners, includingBcl-2 homologous antagonist/killer, Bcl-2-like protein 11 and tumor necorosis factor ligand superfamily member 12, was additionally documented (17). miR-137, as possible negative component of gastric tumorigenesis, suppressed GC cell survival by inducing early apoptosis through targeting Akt2 and affecting downstream Bcl2-associated agonist of cell death, a pro-apoptotic member of the Bcl-2 family, which functions by selectively dimerizing with pro-survival members, B-cell lymphoma-extra large and Bcl-2 (21). Bcl-2, another substrate of Akt, may be directly regulated by miR-449a and thus transduced its tumor-suppressive effects of inducing tumor cell apoptosis and inhibiting cell growth in gastric adenocarcinoma (60). Other proteins of the PI3K/Akt/mTOR pathway, PIK3CB, downstream tuberous sclerosis 1 (TSC1) and mTOR have been demonstrated to be negatively modulated by miR-125b-2, miR-451a and miR-101-2, respectively, which accounted for their inhibitory effects on GC growth, including blocking cell proliferation and colony formation, and inducing cell death (61). Furthermore, upstream RTKs PDGFR and hepatocyte growth factor receptor, receiving platelet-derived growth factor and hepatocyte growth factors respectively, were additionally targeted by miR-34a and mediated its supression on GC cell proliferation via the PI3K/Akt pathway (18). 


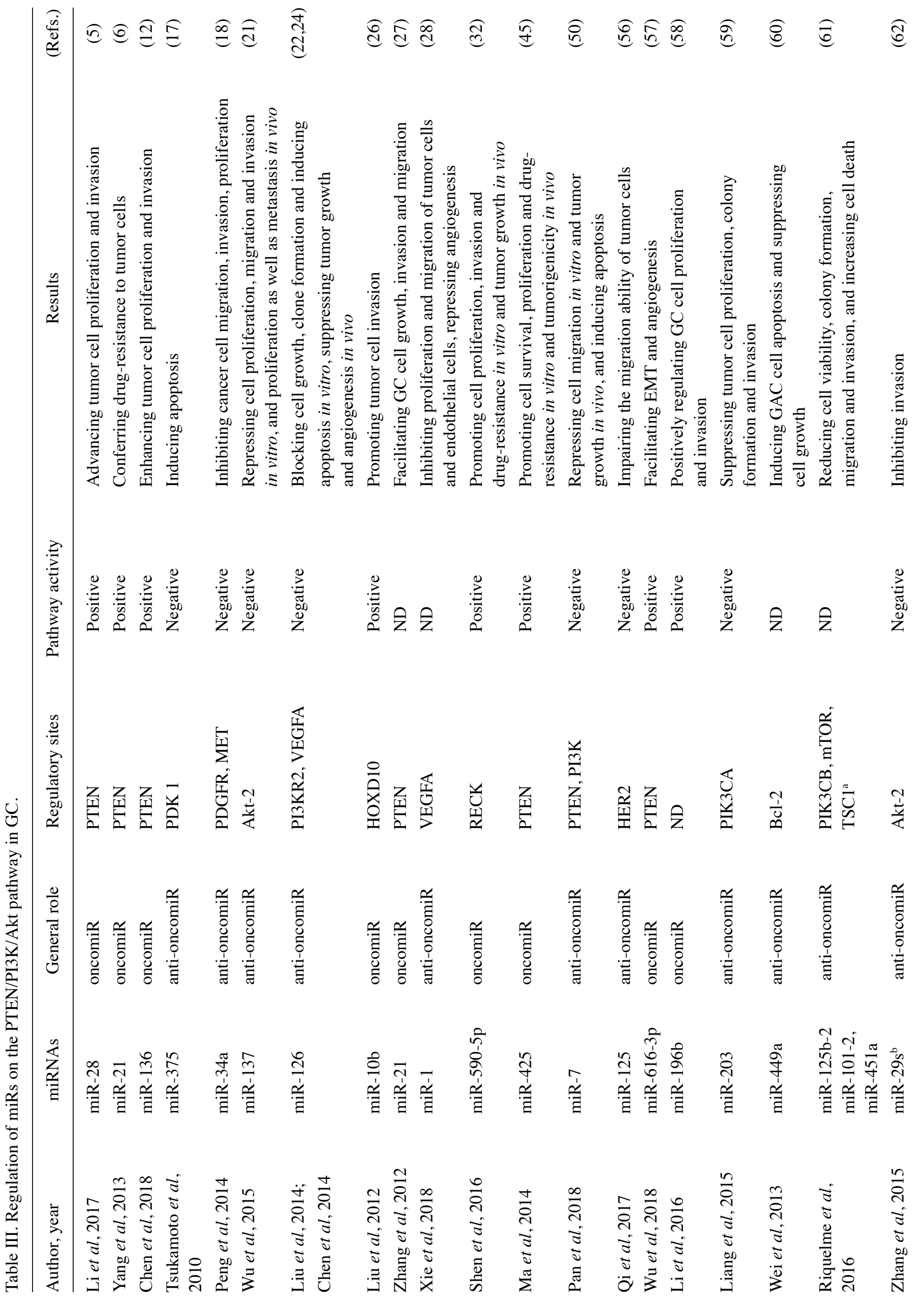




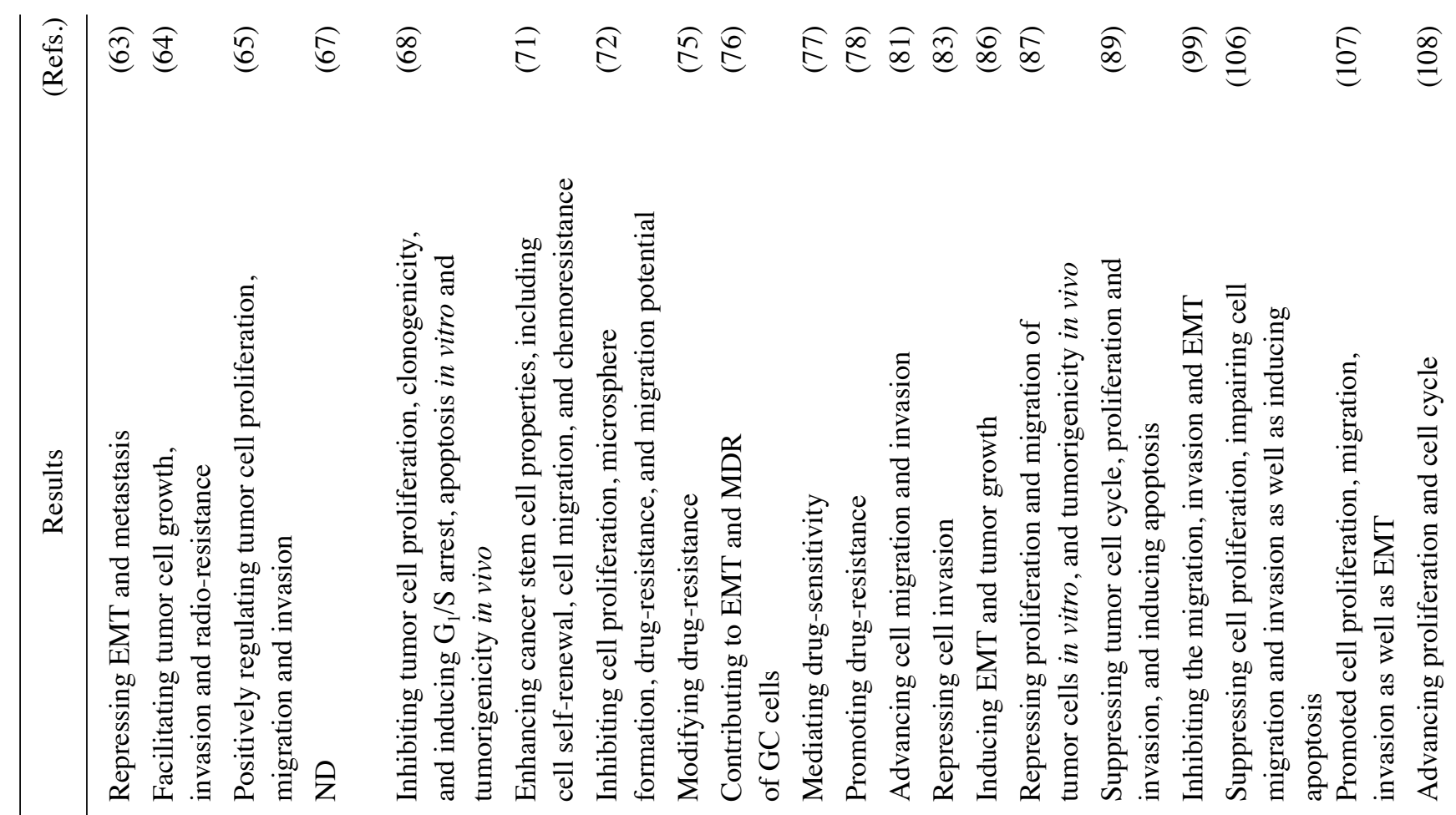

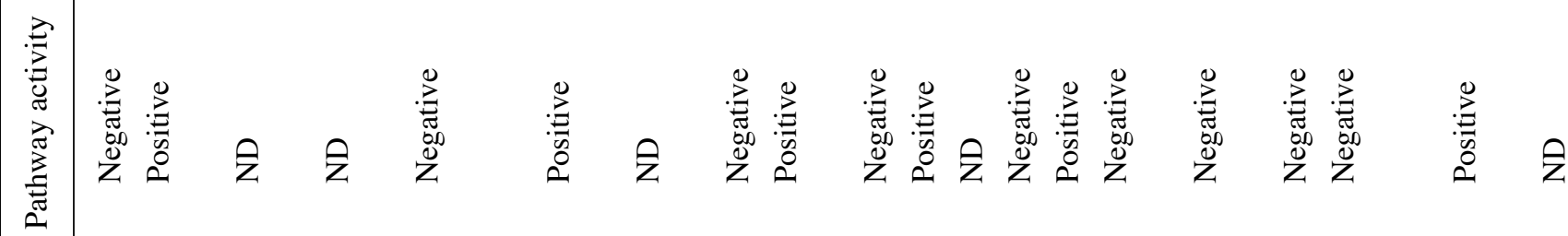

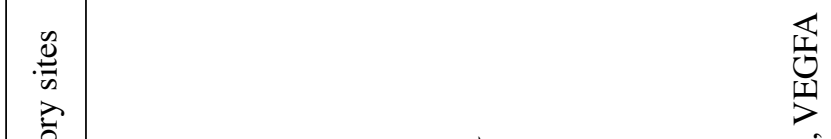

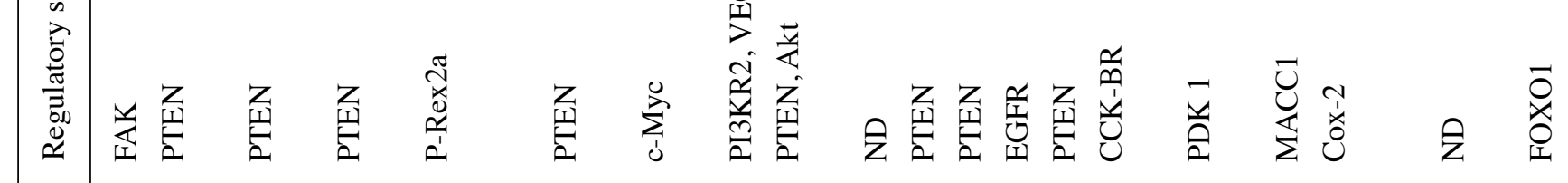

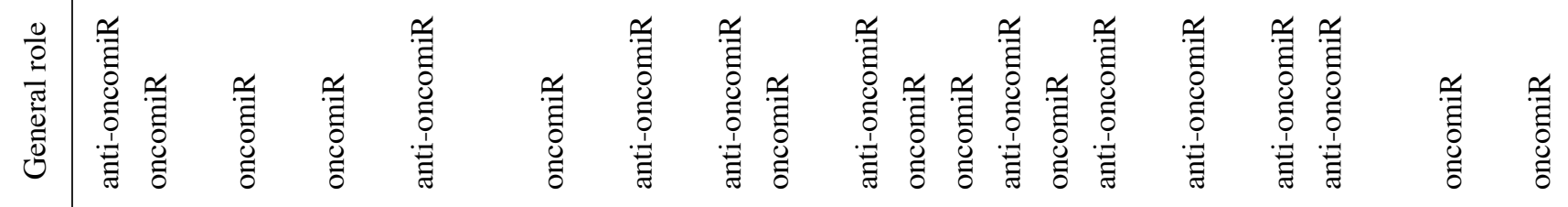

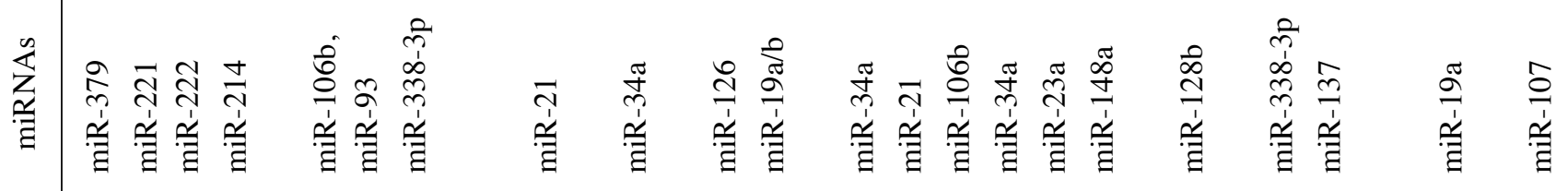

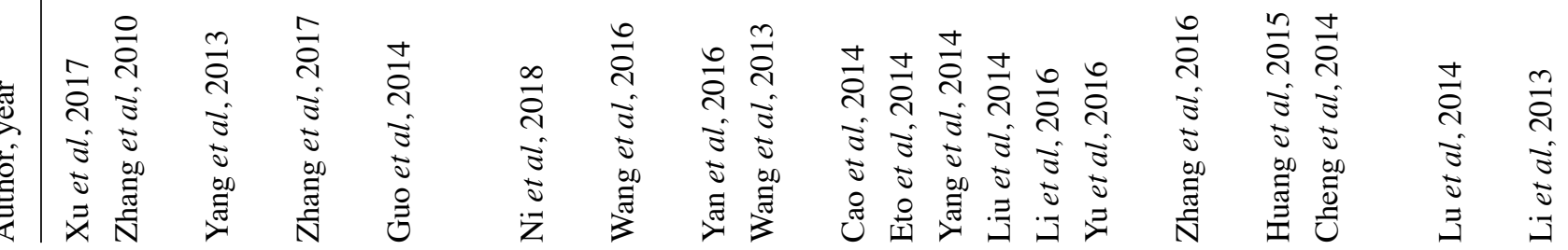


In addition to the involvement in the formation of GC tumors, the regulation of PI3K/Akt signaling by miRNAs is involved in oncogenic activities responsible for GC progression. The miR-29 family, consisting of miR-29a, miR-29b and miR-29c, was able to restrain Akt2 expression and subsequently inactivate GSK3 $\beta$, leading to the impaired invasive ability of GC cells (62). Focal adhesion kinase (FAK) is a crucial transducer of integrin-mediated signaling to downstream pathways, including the PI3K/Akt pathway (63). The activated FAK by integrins may form a binding site for the SH2 domain of p85 subunit and phosphorylate it, which may subsequently activate the p110 catalytic subunit of PI3K (63). Xu et al (63) identified that miR-379 inactivated Akt signaling by suppressing FAK expression, thus leading to inhibition of cell migration, invasion and EMT process. Cullin 4B, a scaffold protein, directly binds to the $\mathrm{S} 2$ region of the miR-125a promoter and transcriptionally represses miR-125a, through which it promotes HER2 expression, thus stimulating downstream PI3K/Akt signaling and the migration of GC cells. In addition to EMT, the activated $\mathrm{Akt} / \mathrm{mTOR}$ signaling is an underlying mechanism of the induced angiogenesis by miR-616-3p in GC (57). Conversely, the reduced activation of Akt/mTOR signaling partially mediated the suppression of miR-126 on GC tumor growth and angiogenesis (24).

\section{miRNAs target and regulate PTEN in GC}

miRNAs are involved in PTEN downregulation. As mentioned above, genetic and epigenetic alteration of PTEN may lead to its downregulation in GC. A number of overexpressed miRNAs may additionally deregulate PTEN by directly combining to the 3'-UTR of its mRNA and thus silencing PTEN at the post-transcriptional level, which is considered another form of epigenetic modification. Chun-Zhi et al (64) demonstrated that miR-221 and miR-222 were upregulated in GC and possessed the ability to target PTEN. Furthermore, ectopic expression of miR-221 and miR-222 in GC cells led to a decreased expression level of PTEN (64). The silenced expression of PTEN in GC may additionally be due to other miRNAs negatively regulating it, including miR-136, miR-214 and miR-28 $(5,12,65)$. Furthermore, the downregulated PTEN expression level was associated with adverse clinical parameters, including lymph node metastasis, poor differentiation and advanced TNM stage of patients with GC (65-67). Different from directly targeting PTEN, the tumor suppressor miR-338-3p was able to indirectly upregulate the activity of PTEN without altering its expression, by suppressing phosphatidylinositol 3,4,5-trisphosphate RAC exchanger 2a, a guanine nucleotide exchange factor for the RAC guanosine triphosphatase (GTPase), which stimulated PI3K signaling by serving as a PTEN-interacting protein and antagonizing it (68).

PTEN mediates oncogenic activities of miRNAs via PI3K/Akt signaling. Due to the critical negative regulator role of PTEN in PTEN/PI3K/Akt signaling, the suppression of PTEN by oncogenic miRNAs results in increased activity of downstream PI3K signaling, and consequently contributed to the malignant phenotypes of GC, including the promoted cell proliferation and survival, impaired drug-sensitivity, and enhanced invasiveness, metastasis and angiogenesis (Table III and Fig. 1). Among these miRNAs, miR-21 is significant. Zhang et al (27) demonstrated that miR-21 promoted the growth, invasion and 


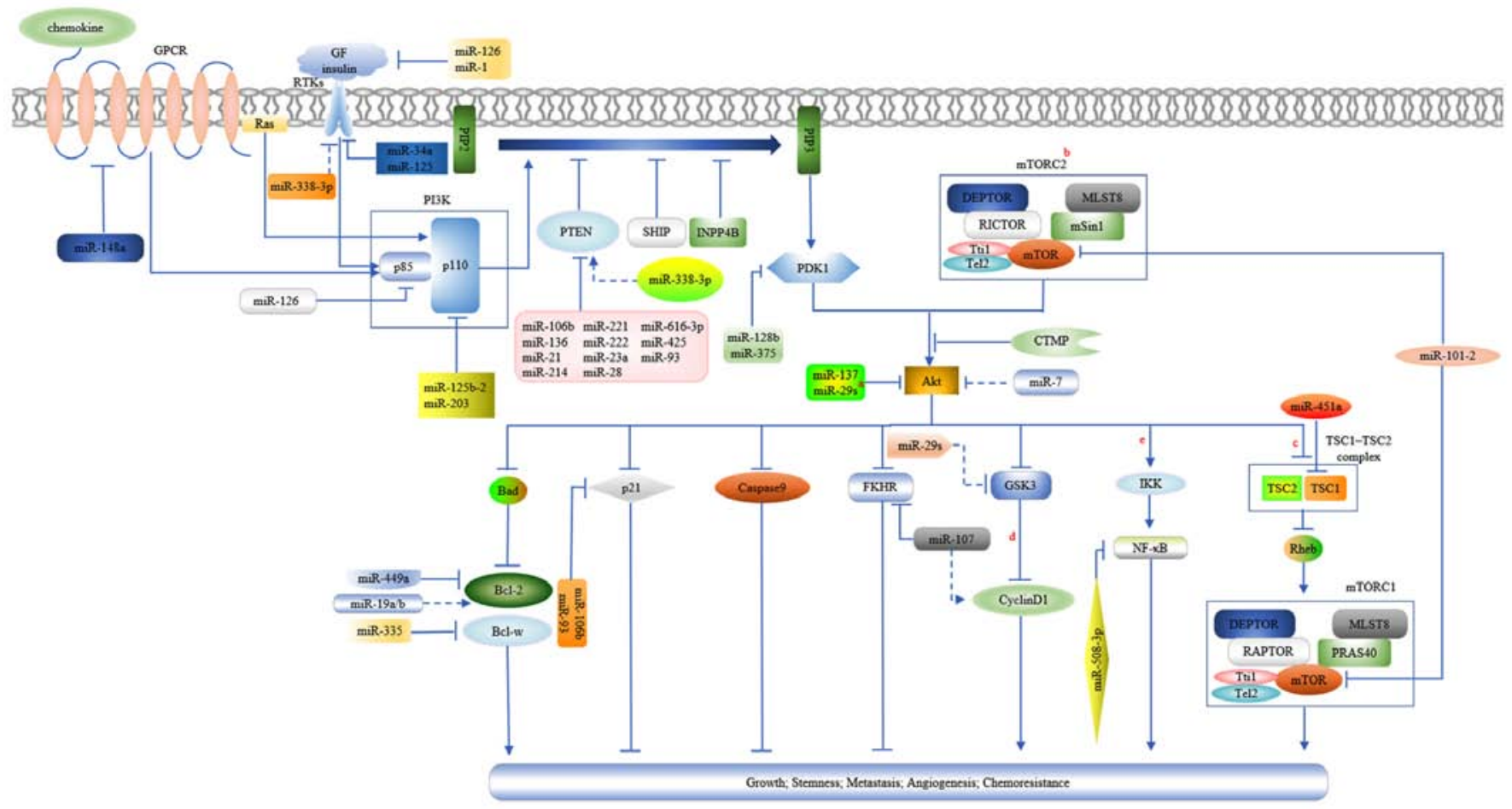

Figure 1. A diagram presenting the activation of the PTEN/PI3K/Akt signaling pathway and the regulations of miRNAs on it in GC. a, miR-29s include miR-29a, miR-29b and miR-29c. b, AKT may promote protein synthesis and cell growth by disintegrating the TSC1/TSC 2 complex by phosphorylating TSC2.c, TSC1/TSC2 complex is a negative regulator of mTORC1 due to its GTPase-activating protein activity for the Rheb GTPase (a member of the Ras family), which may activate mTORC1. mTORC1 is involved in protein and lipid synthesis and anti-autophagy, thus promoting cell growth and proliferation. mTORC1 and mTORC2 possess the mTOR kinase, the scaffolding protein MLST8, the mTOR regulatory subunit DEPTOR, and the Tti1/Tel2 complex, which is critical for mTOR complex assembly and stability. The scaffolding protein RAPTOR and activity inhibitor PRAS40 are specific components belonging to mTORC1. Similarly, mTORC2 has its specific negative regulators, mSin1 and mTOR associated protein RICTOR $(38,94,95)$. d, Inhibition of GSK3 by Akt leads to the accumulation and nuclear translocation of cytoplasmic $\beta$-catenin, which may induce the expression of cyclin D1 through its combination with transcription factors in the nucleus. The increased expression of cyclin D1 facilitates cell cycle progression. Furthermore, the decreased phosphorylation of cyclin D1 from GSK3 inhibition enhances its stabilization (14). e, Akt stimulates IKK by phosphorylation and subsequently, IKK activates NF- $\mathrm{B}$ by phosphorylating the inhibitor of $\kappa \mathrm{B}$, which may mask

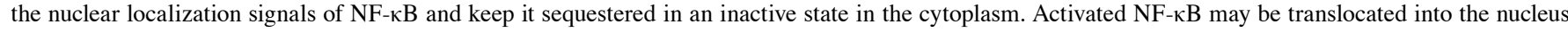
where it transcriptionally upregulates multiple genes, including positive cell-cycle regulators, anti-apoptotic and pro-survival factors $(14,88,96)$. Arrowheads and perpendicular lines indicate stimulation and inhibition of downstream substrates, respectively; straight lines and dotted lines indicate direct and indirect effects on downstream substrates, respectively. Akt, protein kinase B; DEPTOR, DEP domain-containing mTOR-interacting protein; GF, growth factor; GSK3, glycogen synthase kinase 3; GTPase, guanosine triphosphate hydrolase; IKK, inhibitor of nuclear factor $\kappa B$ kinase; miR, microRNA; MLST8, target of rapamycin complex subunit LST8; mSin1, mammalian stress-activated protein kinase interacting protein 1; mTOR, mammalian target of rapamycin; mTORC1, mTOR complex 1;

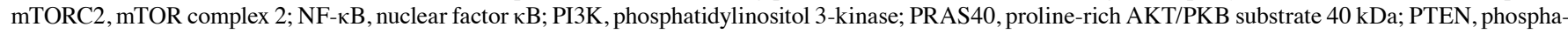
tase and tensin homolog; RAPTOR, regulatory-associated protein of mTOR; Rheb, guanosine triphosphate-binding protein Rheb; RICTOR, rapamycin-insensitive companion of mTOR; Tel2, telomere maintenance 2; TSC1, tuberous sclerosis protein 1; TSC2, tuberous sclerosis protein 2; Tti1, Tel2-interacting protein 1.

migration of GC cells by directly binding to the 3'-UTR of PTEN. Whereas antisense oligonucleotides against miR-21 were able to increase PTEN expression and impair downstream PI3K/Akt/mTOR signaling, leading to reverse effects on these biological behaviors of GC cells $(27,69)$.

In addition, miR-21 was involved in the regulation of a number of Polycomb group (PcG) proteins on gastric cancer stem cells (CSCs), a small subset of cancer cells with self-renewal and tumor-initiating properties, which are critical in tumor metastasis, recurrence and chemoresistance (70). PcG family proteins, including polycomb group RING finger protein 2 (Mel-18), chromobox homolog 7 (CBX7) and enhancer of zeste homolog 2 , form the multi-protein complexes, called polycomb repressive complexes, through which they epigenetically regulate homeotic genes expression by altering chromosomal structure or transcriptional repression (71). In this manner, PcG proteins exert effects in embryonic development, cell proliferation and differentiation, stem cell properties and tumorigenesis (71). Ni et al (71) demonstrated that in addition to inhibiting p16, $\mathrm{CBX} 7$ was additionally able to upregulate miR-21and thus enhance its downstream PTEN/Akt/p53 signaling, whereby it potentiated stem cell-like properties of GC cells, including augmented self-renewal and cell migration, enhanced chemoresistance and increased expression of stem cell markers (CD44 antigen and POU domain, class 5, transcription factor 1). B cell-specific Moloney murine leukemia virus integration site 1 (Bmi-1) is another significant PcG family member, which positively regulates stem cell-like phenotypes of GC, and similarly, it contributed to the CSCs characteristics via the miR-21/PTEN/Akt axis (72). However, Bmi-1 was additionally able to directly inhibit the expression of PTEN and thus activate Akt signaling (72). miR-34a, which negatively regulated CSCs phenotypes, was upregulated by Bmi-1. Notably, miR-34a was able to conversely inhibit Bmi-1 by targeting c-Myc, which increased the expression of Bmi-1 by binding to its promoter, and this reciprocal modulation between Bmi-1 and miR-34a constitute a negative feedback loop in maintaining the stem cell-like properties of GC 
cells (72). Mel-18 was additionally reported to be involved in the regulation of gastric CSCs properties; however, inversely, it exerted negative effects by downregulating the expression of miR-21 and therefore increasing PTEN expression (73).

miRNAs regulate chemosensitivity via the PTEN/PI3K/Akt pathway. miRNAs and PTEN/PI3K/Akt signaling have been significantly implicated in the occurrence of drug-resistance in GC $(11,74)$. Therefore, elucidating the regulation of PTEN/PI3K/Akt pathway by miRNAs involved in the decreased response to chemotherapies may help to clarify the underlying mechanisms.

There are numerous identified causes responsible for tumor chemo-resistance, including intracellular drug efflux, failure to undergo apoptosis, target gene alteration, drug or metabolite detoxification and DNA repair enhancement, among which, the increased drug efflux is a most common one, involving a family of ATP-dependent efflux pumps, termed ATP-binding cassette (ABC) transporters (6,74). Consistently, the enhanced resistance of tumor cells to DDP resulted from overexpression of the $\mathrm{ABC}$ transporter, and multidrug resistance-associated protein 1 was observed in GC, which was induced by the attenuated repression of PI3K/Akt pathway due to downregulated miR-126 expression (75). Wang et al (76) demonstrated that miR-19a/b modulated multidrug resistance by targeting PTEN, which may be partly attributed to the accelerated efflux through miR-19a/b induced upregulation of permeability glycoprotein, another primary member of the $A B C$ transporter. The increased Bcl-2, together with the decreased apoptosis regulator BAX and caspase-3, in GC cells transfected with miR-19a/b represented reduced susceptibility to drug-induced apoptosis, which is another pivotal mechanism underlying chemoresistance as anticancer agents function by inducing tumor cells apoptosis (76). The activated PI3K pathway frequently transduces survival signals in GC $(11,76)$. Survivin, the smallest mammalian member of the inhibitor of apoptosis gene family, inhibits apoptosis initiated by the extrinsic or intrinsic pathways, and it engages in the positive regulation of DDP-induced GC cell apoptosis by miR-34a through PI3K/Akt signaling (77). In addition to conventional chemotherapeutic drugs, suppression of apoptosis induced by overexpressed miR-21 via the PTEN/PI3K/Akt pathway may additionally account for the resistance to trastuzumab of HER2-positive GC cells (78). Consistent with the prediction that miRNAs regulate drug-resistance by interfering with specific therapeutic targets, miR-125a, which targets HER2 and inhibits its downstream Akt signaling, is able to increase the efficacy of trastuzumab when used in combination with suppressing GC cell proliferation (74,79). CSCs are considered a cause of tumor relapse following successful chemotherapy, owing to their quiescence, DNA repair capacity and ABC-transporter expression (70). miRNA has been reported to regulate resistance to epirubicin and other stem cell-like properties through PTEN/PI3K/AKT signaling in GC $(71,72)$.

In previous studies examining the involvement of PI3K/Akt signaling in GC drug-resistance, inhibitors against this pathway have been frequently employed. Yang et al (6) demonstrated that LY294002 inhibited miR-21-activated PI3K/Akt signaling and consequently promoted cell survival and DDP resistance. Similarly, compared with controls, lower activity of PI3K/Akt signaling while higher percentage of apoptosis cells were documented in DDP incubated GC cells following treatment with miR-34a mimics and wortmannin (77). These results not only suggested the association of this pathway in sensitivity to anticancer drugs modulated by miRNAs; however, additionally suggest the potential clinical usage of these small molecule inhibitors in modifying chemotherapy efficacy for patients with GC.

miRNA/PTEN axis is involved in gastric tumor microenvironment. During cancer progression, a co-evolving and supportive environment termed the tumor microenvironment is important, which consists of ECM adjacent to cancer cells and non-malignant stromal cells, including fibroblasts, endothelial cells and hematopoietic cells, primarily macrophages and lymphocytes (2). The crosstalk between cancer cells and the environment may have substantial impacts on tumor development, including tumor growth, metastasis and refractoriness to therapies, wherein the regulation of PTEN/PI3K/Akt by miRNAs may be involved $(2,80)$. Yang et al (81) identified four upregulated miRNAs and seven downregulated miRNAs in gastric cancer associated fibroblasts (CAFs), the most abundant cells in the tumor microenvironment, compared with normal fibroblasts. CAFs promoted the growth and metastasis of tumor cells, and silencing miR-106b in CAFs abolished the contributive effect on cell motility due to PTEN upregulation (81). Similarly, when cultured in conditioned medium derived from miR-1-transfected GC cells, microvascular endothelial cells were prevented from migrating and forming novel vessels, which was associated with the decreased VEGF-A and endothelin 1 (28). Zheng et al (2) observed that miR-21 in exosomes secreted from tumor-associated macrophages (TAMs) may be ingested by GC cells, and led to impaired sensitivity to DDP via the PTEN/PI3K/Akt pathway. Under low glucose condition, the energy-responding miR-451 may be transferred from GC cells to infiltrated $\mathrm{T}$ cells through exosomes, thus enhancing the differentiation of T-helper 17, wherein the upregulated mTOR signaling induced by miR-451 was involved (30).

The bidirectional impact between cancer cells and tumor microenvironment may additionally be fulfilled by the secreted soluble factors, including cytokines, chemokines and growth factors in autocrine or paracrine manner (80). Ma et al (45) demonstrated that when exposing GC cells to pro-inflammatory cytokine interleukin- $1 \beta$, miR- 425 was transcriptionally induced, and thus the PTEN expression level was decreased, leading to promoted cell survival and proliferation in vitro in addition to tumor growth in vivo. EMT of GC cells induced by TGF- $\beta 1$ may be stimulated by miR- 21 through its negative regulation of PTEN and the subsequent activation of PI3K/Akt signaling (66). MMPs, a family of zinc-dependent proteases released by tumor cells and TAMs, were implicated in numerous oncogenic processes, including ECM degradation, tumor cell migration and the release of growth factors sequestered within the ECM (82). A previous study demonstrated that MMP-7 was regulated by miR-34a through EGFR/PI3K/Akt signaling during GC progression (83).

Other ncRNAs in co-regulation of the PTEN/PI3K/Akt pathway. ncRNAs, refer to a type of RNA, which do not encode proteins, primarily including miRNAs, long non-coding 
RNAs (lncRNAs), in addition to pseudogenes and circular RNAs (circRNAs) (84). In the competitive endogenous RNA (ceRNA) hypothesis depicting a post-transcriptional regulation network mediated by miRNAs, any RNA molecules sharing one or more miRNA response elements, including protein-coding and ncRNAs, are able to compete for binding to miRNAs and subsequently co-regulate expression of one another (9). Due to their complexity, diversity and frequent dysregulation in cancer, ncRNAs as ceRNAs have attracted increasing attention in oncogenesis and development, regarding the novel layer of post-transcriptional regulation on miRNA-targeted mRNAs provided by them (84).

HOX transcript antisense intergenic RNA (HOTAIR), a lncRNA associated with Polycomb repressive complex 2, was demonstrated to be capable of functioning as a ceRNA of miR-126 to promote expression of miR-126 target genes VEGF-A and PI3KR2, resulting in the activation of PI3K/Akt signaling, which was underlying the DDP-resistance induced by overexpressed HOTAIR in GC (75). Pseudogenes are segments of DNA associated with real genes, which are frequently derived from accumulated mutations in a gene and thus commonly lose specific functionality compared with the complete gene (85). Phosphatase and tensin homolog, pseudogene 1, was reported to serve as a tumor suppressor of GC by inhibiting tumor growth and metastasis, whereby it upregulated the expression of its cognate gene PTEN, by sponging miR-106b and miR-93 $(67,84)$. circRNAs are another class of non-coding RNAs, with the $3^{\prime}$ and 5' ends of RNA molecules joined together to form a covalently closed continuous loop. Pan et al (50) identified that the circRNA sponge for miR-7, was able to antagonize the effects of miR-7 on the PTEN/PI3K/Akt pathway, and reverse its repression of tumor cell migration and colony formation in vitro and GC genesis in vivo.

miRNAs synergistically modulate the PI3K cascade with other pathways. The complex crosstalk and pathway convergence among different signaling pathways ensure that no pathway operates in isolation; however, miRNAs exert extensive regulation and a single miRNA may regulate multiple targets due to its imperfect complementary pairing with mRNAs. It is therefore rational to address that miRNAs may regulate PTEN/PI3K/Akt signaling with other crucial pathways co-operatively. Indeed, this has been validated in the pathologic process of GC.

The complex associations between the PI3K/Akt pathway and the Ras/Raf proto-oncogene serine/threonine-protein kinase (Raf)/mitogen-activated protein kinase (MAPK) kinase/MAPK pathway have been well documented, including the inhibition of the extracellular-regulated kinase (ERK) pathway by Akt via its direct phosphorylation of c-Raf on T259, and similarly, the phosphorylation of Akt on amino acid residue $\mathrm{S} 83$ of apoptosis signal-regulated kinase 1, which leads to the impaired activation of the c-Jun $\mathrm{N}$-terminal kinase and p38 pathways $(4,15)$. Ras was additionally able to function upstream of PI3K by interacting with the RBD of p110 catalytic subunit and thus activating PI3K signaling (39). In $\mathrm{GC}, \mathrm{Li}$ et al (86) demonstrated that the activation of Akt in addition to the ERK pathways accounted for miR-23a-induced EMT. It was observed that miR-126 repressed angiogenesis by simultaneously inhibiting downstream Akt/mTOR and
ERK signaling (24). In addition to Akt and ERK signaling, the activation of the signal transducer and activator of transcription 3 (STAT3) pathway was involved in the oncogenic activities induced by miR-590-5p, including the promotion of cell proliferation, invasion, drug-resistance and tumor growth (32). Conversely, the negative regulation of Akt and STAT3 signaling by targeting the GPCR for gastrin and cholecystokinin may be the underlying mechanism for the tumor-suppressive role of miR-148a in GC (87).

In GC cells, it has been suggested that the activation of PI3K or loss of PTEN was able to protect integrin-linked kinase (ILK) from proteasome-mediated degradation (88). Enhanced ILK may subsequently activate Ras and promote the formation of the IQ motif-containing GTPase-activating protein 1-Ras complex, which stimulated the c-Raf/MEK1/2/ERK1/2/ribosomal S6 kinase/inhibitor of $\kappa B \alpha /$ nuclear factor (NF)- $\kappa B$ signaling, leading to the increased cell growth, migration and decreased sensitivity of 5-FU and DDP (88). Consistently, miR-128b was reported to repress GC cell growth, invasion and promote apoptosis through $\mathrm{Akt} / \mathrm{NF}-\mathrm{\kappa B}$ signaling due to its negative post-transcriptional regulation of PDK1 (89). Furthermore, PI3K/Akt/NF- $\mathrm{KB}$ signaling inactivation was a possible mechanism of the pro-apoptosis effect of celastrol, an antitumor plant triterpene with the ability to inhibit miR-21 expression (90). Apart from serving roles downstream of the PI3K/Akt pathway, NF- $\mathrm{KB}$ was additionally able to affect the expression of PTEN and downstream Akt signaling, mediated by its transcriptional regulation of miRNAs, including miR-425 and miR-21 (45,71). Notably, the increased NF- $\kappa B$ transcriptional activity may be due to the upregulated Akt activation (45,71).

\section{Conclusion}

In the last decade or two, the close association between miRNA dysregulation and GC has been well documented. In previous studies investigating the mechanisms by which miRNAs are involved in gastric tumorigenicity and progression, the results demonstrated that dysregulated miRNAs exerted their promotive effects by post-transcriptionally regulating oncogenes and tumor-suppressors, and thus affecting associated canonical pathways, among which PTEN/PI3K/Akt was a critical one due to its decisive role in GC tumor growth, metastasis, angiogenesis, stemness and chemoresistance.

Identifying molecular causes of GC is of great importance in pattern recognition and therapeutic strategies. The poor prognosis of patients with GC primarily results from late detection, aggressive progression, poor response to available therapies and relapse $(6,8,49)$. Therefore, in the present review, it was discussed how miRNAs are involved in the regulation of gastric CSCs and tumor environment through PTEN/PI3K/Akt signaling, which bears resemblance to the 'seed' and 'soil' in GC pathogenesis (91), respectively. Similar investigations have additionally been summarized regarding sensitivity to conventional chemotherapies in addition to target therapies against this tumor. Based on these molecular mechanisms, the potential applications of miRNAs as novel biomarkers for diagnosis and prognosis-prediction were discussed. As drugs (including miRNA mimics and inhibitors) have been tested in preclinical and clinical trials of a number of diseases (92), the 
role of miRNAs as promising therapeutic targets for GC was additionally discussed. Besides, inhibitors or monoclonal antibodies against the PTEN/PI3K/Akt pathway and its up/down stream molecules have been in different phases of clinical trial or in applications for GC. Furthermore, it has been reported that ectopic expression of miRNA in combination with a PI3K/Akt pathway inhibitor may acquire synergistic treatment efficacy (93). Therefore, further investigations into miRNAs and PTEN/PI3K/Akt signaling in addition to their interactions are necessary and rewarding for improving the clinical management and outcome of patients with GC.

\section{Acknowledgements}

Not applicable.

\section{Funding}

The present study was supported by funding from National Science Foundation Grants of China (grant nos. 81160307 and 81560395), the Jiangxi Science \& Technology Pillar Program and the Science Foundation for Young Scholars of Jiangxi Province (grant no. 2007GQY1167).

\section{Availability of data and materials}

Data sharing is not applicable to this article, as no datasets were generated or analyzed during the current study.

\section{Authors' contributions}

MH and SZ wrote the manuscript. SX and XX constructed the tables and diagrams. MH, SZ, SX, XX and XZ checked and revised the manuscript and were involved in the conception of the study. Additionally, XZ was responsible for the organization, revision and submission of this manuscript. All authors read and approved the final manuscript.

\section{Ethics approval and consent to participate}

Not applicable.

\section{Patient consent for publication}

Not applicable.

\section{Competing interests}

The authors declare that they have no competing interests.

\section{References}

1. Pan HW, Li SC and Tsai KW: MicroRNA dysregulation in gastric cancer. Curr Pharm Des 19: 1273-1284, 2013.

2. Zheng P, Chen L, Yuan X, Luo Q, Liu Y, Xie G, Ma Y and Shen L: Exosomal transfer of tumor-associated macrophage-derived miR-21 confers cisplatin resistance in gastric cancer cells. J Exp Clin Cancer Res 36: 53, 2017.

3. Zhou X, Men X, Zhao R, Han J, Fan Z, Wang Y, Lv Y, Zuo J, Zhao L, Sang M, et al: miR-200c inhibits TGF- $\beta$-induced-EMT to restore trastuzumab sensitivity by targeting ZEB1 and ZEB2 in gastric cancer. Cancer Gene Ther 25: 68-76, 2018.
4. Zhang Z, Li Z, Li Y and Zang A: MicroRNA and signaling pathways in gastric cancer. Cancer Gene Ther 21: 305-316, 2014.

5. Li L, Zhu X, Shou T, Yang L, Cheng X, Wang J, Deng L and Zheng Y: MicroRNA-28 promotes cell proliferation and invasion in gastric cancer via the PTEN/PI3K/AKT signalling pathway. Mol Med Rep 17: 4003-4010, 2017.

6. Yang SM, Huang C, Li XF, Yu MZ, He Y and Li J: miR-21 confers cisplatin resistance in gastric cancer cells by regulating PTEN. Toxicology 306: 162-168, 2013.

7. Petrocca F, Visone R, Onelli MR, Shah MH, Nicoloso MS de Martino I, Iliopoulos D, Pilozzi E, Liu CG, Negrini M, et al: E2F1-regulated MicroRNAs impair TGFbeta-dependent cell-cycle arrest and apoptosis in gastric cancer. Cancer Cell 13: 272-286, 2008

8. Zhang H, Qu Y, Jingjing D, Deng T, Liu R, Zhang L, Bai M, Li J, Zhou L, Ning T, et al: Integrated analysis of the miRNA, gene and pathway regulatory network in gastric cancer. Oncol Rep 35: 1135-1146, 2016.

9. Zhang JX, Chen ZH, Chen DL, Tian XP, Wang CY, Zhou ZW, Gao Y, Xu Y, Chen C, Zheng ZS, et al: LINC01410miR-532-NCF2-NF- $\kappa$ B feedback loop promotes gastric cancer angiogenesis and metastasis. Oncogene 37: 2660-2675, 2018.

10. Li T, Lu YY, Zhao XD, Guo HQ, Liu CH, Li H, Zhou L, Han YN, Wu KC, Nie YZ, et al: MicroRNA-296-5p increases proliferation in gastric cancer through repression of Caudal-related homeobox 1. Oncogene 33: 783-793, 2014.

11. Matsuoka T and Yashiro M: The role of PI3K/Akt/mTOR signaling in gastric carcinoma. Cancers (Basel) 6: 1441-1463, 2014.

12. Chen $X$, Huang $Z$ and Chen R: Microrna-136 promotes proliferation and invasion ingastric cancer cells through Pten/Akt/P-Akt signaling pathway. Oncol Lett 15: 4683-4689, 2018.

13. Dahia PL: PTEN, a unique tumor suppressor gene. Endocr Relat Cancer 7: 115-129, 2000.

14. Fresno Vara JA, Casado E, de Castro J, Cejas P, Belda-Iniesta C and González-Barón M: PI3K/Akt signalling pathway and cancer. Cancer Treat Rev 30: 193-204, 2004.

15. Manning BD and Cantley LC: AKT/PKB signaling: Navigating downstream. Cell 129: 1261-1274, 2007.

16. Kang YH, Lee HS and Kim WH: Promoter methylation and silencing of PTEN in gastric carcinoma. Lab Invest 82: 285-291, 2002.

17. Tsukamoto Y, Nakada C, Noguchi T, Tanigawa M, Nguyen LT, Uchida T, Hijiya N, Matsuura K, Fujioka T, Seto $M$ and Moriyama M: MicroRNA-375 is downregulated in gastric carcinomas and regulates cell survival by targeting PDK 1 and 14-3-3zeta. Cancer Res 70: 2339-2349, 2010.

18. Peng Y, Guo JJ, Liu YM and Wu XL: MicroRNA-34A inhibits the growth, invasion and metastasis of gastric cancer by targeting PDGFR and MET expression. Bioscience Reports 34: e00112, 2014.

19. Wang T, Hou J, Li Z, Zheng Z, Wei J, Song D, Hu T, Wu Q, Yang JY and Cai JC: miR-15a-3p and miR-16-1-3p negatively regulate twist1 to repress gastric cancer cell invasion and metastasis. Int J Biol Sci 13: 122-134, 2017.

20. Kang W, Tong JHM, Lung RWM, Dong Y, Zhao J, Liang Q, Zhang L, Pan Y, Yang W, Pang JCS, et al: Targeting of YAP1 by microRNA-15a and microRNA-16-1 exerts tumor suppressor function in gastric adenocarcinoma. Mol Cancer 14: 52, 2015.

21. Wu L, Chen J, Ding C, Wei S, Zhu Y, Yang W, Zhang X, Wei X and Han D: MicroRNA-137 contributes to dampened tumorigenesis in human gastric cancer by Targeting AKT2. PLoS One 10: $\mathrm{e} 0130124,2015$.

22. Liu LY, Wang W, Zhao LY, Guo B, Yang J, Zhao XG, Hou N, Ni L, Wang AY, Song TS, et al: miR-126 inhibits growth of SGC-7901 cells by synergistically targeting the oncogenes PI3KR 2 and Crk, and the tumor suppressor PLK2. Int J Oncol 45: 1257-1265, 2014.

23. Feng R, Chen X, Yu Y, Su L, Yu B, Li J, Cai Q, Yan M, Liu B and Zhu Z: miR-126 functions as a tumour suppressor in human gastric cancer. Cancer Lett 298: 50-63, 2010.

24. Chen H, Li L, Wang S, Lei Y, Ge Q, Lv N, Zhou X and Chen C: Reduced miR-126 expression facilitates angiogenesis of gastric cancer through its regulation on VEGF-A. Oncotarget 5: $11873-11885,2014$

25. Otsubo T, Akiyama Y, Hashimoto Y, Shimada S, Goto K and Yuasa Y: MicroRNA-126 inhibits SOX2 expression and contributes to gastric carcinogenesis. PLoS One 6: e16617, 2011.

26. Liu Z, Zhu J, Cao H, Ren H and Fang X: miR-10b promotes cell invasion through RhoC-AKT signaling pathway by targeting HOXD10 in gastric cancer. Int J Oncol 40: 1553-1560, 2012. 
27. Zhang BG, Li JF, Yu BQ, Zhu ZG, Liu BY and Yan MIN MicroRNA-21 promotes tumor proliferation and invasion in gastric cancer by targeting PTEN. Oncol Rep 27: 1019-1026, 2012.

28. Xie M, Dart DA, Guo T, Xing XF, Cheng XJ, Du H, Jiang WG, Wen XZ and Ji JF: MicroRNA-1 acts as a tumor suppressor microRNA by inhibiting angiogenesis-related growth factors in human gastric cancer. Gastric Cancer 21: 41-54, 2018.

29. Kogo R, Mimori K, Tanaka F, Komune S and Mori M: Clinical significance of miR-146a in gastric cancer cases. Clin Cancer Res 17: 4277-4284, 2011.

30. Liu F, Bu Z, Zhao F and Xiao D: Increased T-helper 17 cell differentiation mediated by exosome-mediated microRNA-451 redistribution in gastric cancer infiltrated T cells. Cancer Sci 109: 65-73, 2018.

31. Zhu C, Ren C, Han J, Ding Y, Du J, Dai N, Dai J, Ma H, Hu Z, Shen $\mathrm{H}$, et al: A five-microRNA panel in plasma was identified as potential biomarker for early detection of gastric cancer. $\mathrm{Br}$ J Cancer 110: 2291-2299, 2014.

32. Shen B, Yu S, Zhang Y, Yuan Y, Li X, Zhong J and Feng J: miR-590-5p regulates gastric cancer cell growth and chemosensitivity through RECK and the AKT/ERK pathway. OncoTargets Ther 9: 6009-6019, 2016

33. Zhou X, Ye F, Yin C, Zhuang Y, Yue G and Zhang G: The interaction between miR-141 and lncRNA-H19 in regulating cell proliferation and migration in gastric cancer. Cell Physiol Biochem 36: 1440-1452, 2015.

34. Han TS, Hur K, Xu G, Choi B, Okugawa Y, Toiyama Y, Oshima H, Oshima M, Lee HJ, Kim VN, et al: MicroRNA-29c mediates initiation of gastric carcinogenesis by directly targeting ITGB1. Gut 64: 203-214, 2015

35. Zhao L and Vogt PK: Class I PI3K in oncogenic cellular transformation. Oncogene 27: 5486-5496, 2008.

36. Burgering BM and Coffer PJ: Protein kinase B (c-Akt) in phosphatidylinositol-3-OH kinase signal transduction. Nature 376: 599-602, 1995

37. Martini M, De Santis MC, Braccini L, Gulluni F and Hirsch E: PI3K/AKT signaling pathway and cancer: An updated review. Ann Med 46: 372-383, 2014.

38. Janku F, Yap TA and Meric-Bernstam F: Targeting the PI3K pathway in cancer: Are we making headway? Nat Rev Clin Oncol 15: 273-291, 2018.

39. Yang HW, Shin MG, Lee S, Kim JR, Park WS, Cho KH, Meyer T and Heo WD: Cooperative activation of PI3K by Ras and Rho family small GTPases. Mol Cell 47: 281-290, 2012.

40. Samuels Y and Ericson K: Oncogenic PI3K and its role in cancer. Curr Opin Oncol 18: 77-82, 2006.

41. Sarbassov DD, Guertin DA, Ali SM and Sabatini DM: Phosphorylation and regulation of Akt/PKB by the Rictor-mTOR complex. Science 307: 1098-1101, 2005.

42. Soung YH, Lee JW, Nam SW, Lee JY, Yoo NJ and Lee SH Mutational analysis of AKT1, AKT2 and AKT3 genes in common human carcinomas. Oncology 70: 285-289, 2006

43. Maira SM, Galetic I, Brazil DP, Kaech S, Ingley E, Thelen M and Hemmings BA: Carboxyl-terminal modulator protein (CTMP), a negative regulator of $\mathrm{PKB} / \mathrm{Akt}$ and $\mathrm{v}$-Akt at the plasma membrane. Science 294: 374-380, 2001.

44. Li VS, Wong CW, Chan TL, Chan AS, Zhao W, Chu KM, So S, Chen X, Yuen ST and Leung SY: Mutations of PIK3CA in gastric adenocarcinoma. BMC Cancer 5: 29, 2005.

45. Ma J, Liu J, Wang Z, Gu X, Fan Y, Zhang W, Xu L, Zhang J and Cai D: NF-kappaB-dependent MicroRNA-425 upregulation promotes gastric cancer cell growth by targeting PTEN upon IL-1 $\beta$ induction. Mol Cancer 13: 40, 2014.

46. Wen YG, Wang Q, Zhou CZ, Qiu GQ, Peng ZH and Tang HM: Mutation analysis of tumor suppressor gene PTEN in patients with gastric carcinomas and its impact on PI3K/AKT pathway. Oncol Rep 24: 89-95, 2010.

47. Samuels Y, Wang Z, Bardelli A, Silliman N, Ptak J, Szabo S, Yan H, Gazdar A, Powell SM, Riggins GJ, et al: High frequency of mutations of the PIK3CA gene in human cancers. Science 304: $554,2004$.

48. Shi J, Yao D, Liu W, Wang N, Lv H, Zhang G, Ji M, Xu L, He N, Shi B and Hou P: Highly frequent PIK3CA amplification is associated with poor prognosis in gastric cancer. BMC Cancer 12: $50,2012$.

49. Xiong J, Li Z, Zhang Y, Li D, Zhang G, Luo X, Jie Z, Liu Y, Cao Y, Le Z, et al: PRL-3 promotes the peritoneal metastasis of gastric cancer through the PI3K/Akt signaling pathway by regulating PTEN. Oncol Rep 36: 1819-1828, 2016.
50. Pan H, Li T, Jiang Y, Pan C, Ding Y, Huang Z, Yu H and Kong D: Overexpression of circular RNA ciRS-7 abrogates the tumor suppressive effect of miR-7 on gastric cancer via PTEN/PI3K/AKT signaling pathway. J Cell Biochem 119: 440-446, 2018.

51. Zhao S, Wang L, Zhang C, Deng Y, Zhao B, Ren Y, Fu Y and Meng X: Inhibitor of growth 3 induces cell death by regulating cell proliferation, apoptosis and cell cycle arrest by blocking the PI3K/AKT pathway. Cancer Gene Ther 25: 240-247, 2018.

52. Wang SQ, Wang C, Chang LM, Zhou KR, Wang JW, Ke Y, Yang DX, Shi HG, Wang R, Shi XL, et al: Geridonin and paclitaxel act synergistically to inhibit the proliferation of gastric cancer cells through ROS-mediated regulation of the PTEN/PI3K/Akt pathway. Oncotarget 7: 72990-73002, 2016.

53. Li C, Zhang J, Wu H, Li L, Yang C, Song S, Peng P, Shao M, Zhang M, Zhao J, et al: Lectin-like oxidized low-density lipoprotein receptor-1 facilitates metastasis of gastric cancer through driving epithelial-mesenchymal transition and PI3K/Akt/GSK3 $\beta$ activation. Sci Rep 7: 45275, 2017.

54. Chen D, Lin X, Zhang C, Liu Z, Chen Z, Li Z, Wang J, Li B, Hu Y, Dong B, et al: Dual PI3K/mTOR inhibitor BEZ235 as a promising therapeutic strategy against paclitaxel-resistant gastric cancer via targeting PI3K/Akt/mTOR pathway. Cell Death Dis 9: 123, 2018.

55. Jin P and Sun L: Tu1968 the effect of a novel Akt inhibitor MK-2206 on proliferation and chemosensitivity of gastric cancer. Gastroenterology 148 (Suppl 1): S948-S949, 2015.

56. Qi M, Jiao M, Li X, Hu J, Wang L, Zou Y, Zhao M, Zhang R, Liu H, Mi J, et al: CUL4B promotes gastric cancer invasion and metastasis-involvement of upregulation of HER2. Oncogene 37: 1075-1085, 2017.

57. Wu ZH, Lin C, Liu CC, Jiang WW, Huang MZ, Liu $X$ and Guo WJ: miR-616-3p promotes angiogenesis and EMT in gastric cancer via the PTEN/AKT/mTOR pathway. Biochem Biophys Res Commun 501: 1068-1073, 2018.

58. Li NA, Wang W, Xu B and Gong H: miR-196b regulates gastric cancer cell proliferation and invasion via PI3K/AKT/mTOR signaling pathway. Oncol Lett 11: 1745-1749, 2016.

59. Liang M, Shi B, Liu J, He L, Yi G, Zhou L, Yu G and Zhou X: Downregulation of miR203 induces overexpression of PIK3CA and predicts poor prognosis of gastric cancer patients. Drug Des Devel Ther 9: 3607-3616, 2015.

60. Wei B, Song Y, Zhang Y and Hu M: microRNA-449a functions as a tumor-suppressor in gastric adenocarcinoma by targeting Bcl-2. Oncol Lett 6: 1713-1718, 2013.

61. Riquelme I, Tapia O, Leal P, Sandoval A, Varga MG, Letelier P, Buchegger K, Bizama C, Espinoza JA, Peek RM, et al: miR-101-2, miR-125b-2 and miR-451a act as potential tumor suppressors in gastric cancer through regulation of the PI3K/AKT/mTOR pathway. Cell Oncol (Dordr) 39: 23-33, 2016.

62. Zhang H, Cheng Y, Jia C, Yu S, Xiao Y and Chen J: MicroRNA-29s could target AKT2 to inhibit gastric cancer cells invasion ability. Med Oncol 32: 342, 2015.

63. Xu M, Qin S, Cao F, Ding S and Li M: MicroRNA-379 inhibits metastasis and epithelial-mesenchymal transition via targeting FAK/AKT signaling in gastric cancer. Int J Oncol 51: 867-876, 2017.

64. Chun-Zhi Z, Lei H, An-Ling Z, Yan-Chao F, Xiao Y, Guang-Xiu W,Zhi-Fan J, Pei-Yu P, Qing-Yu Z and Chun-Sheng K: MicroRNA-221 and microRNA-222 regulate gastric carcinoma cell proliferation and radioresistance by targeting PTEN. BMC Cancer 10: 367, 2010.

65. Yang TS, Yang XH, Wang XD, Wang YL, Zhou B and Song ZS: miR-214 regulate gastric cancer cell proliferation, migration and invasion by targeting PTEN. Cancer Cell Int 13: 68, 2013.

66. Li C, Song L, Zhang Z, Bai XX, Cui MF and Ma LJ: MicroRNA-21 promotes TGF- $\beta 1$-induced epithelial-mesenchymal transition in gastric cancer through up-regulating PTEN expression. Oncotarget 7: 66989-67003, 2016.

67. Zhang R, Guo Y, Ma Z, Ma G, Xue Q, Li F and Liu L: Long non-coding RNA PTENP1 functions as a ceRNA to modulate PTEN level by decoying miR-106b and miR-93 in gastric cancer. Oncotarget 8: 26079-26089, 2017.

68. Guo B, Liu L, Yao J, Ma R, Chang D, Li Z, Song T and Huang C: miR-338-3p suppresses gastric cancer progression through a PTEN-AKT axis by targeting P-REX2a. Mol Cancer Res 12: 313-321, 2014.

69. Wang P, Guan Q, Zhou D, Yu Z, Song Y and Qiu W: miR-21 inhibitors modulate biological functions of gastric cancer cells via PTEN/PI3K/mTOR pathway. DNA Cell Biol 37: 38-45, 2018.

70. Dean M, Fojo T and Bates S: Tumour stem cells and drug resistance. Nat Rev Cancer 5: 275-284, 2005. 
71. Ni SJ, Zhao LQ, Wang XF, Wu ZH, Hua RX, Wan $\mathrm{CH}$, Zhang JY, Zhang XW, Huang MZ, Gan L, et al: CBX7 regulates stem cell-like properties of gastric cancer cells via p16 and AKT-NF-кB-miR-21 pathways. J Hematol Oncol 11: 17, 2018.

72. Wang X, Wang C, Zhang X, Hua R, Gan L, Huang M, Zhao L, Ni S and Guo W: Bmi-1 regulates stem cell-like properties of gastric cancer cells via modulating miRNAs. J Hematol Oncol 9: 90, 2016.

73. Wang XF, Zhang XW, Hua RX, Du YQ, Huang MZ, Liu Y, Cheng YF and Guo WJ: Mel-18 negatively regulates stem cell-like properties through downregulation of miR-21 in gastric cancer. Oncotarget 7: 63352-63361, 2016.

74. Li H and Yang BB: Friend or foe: The role of microRNA in chemotherapy resistance. Acta Pharmacol Sin 34: 870-879, 2013.

75. Yan J, Dang Y, Liu S, Zhang Y and Zhang G: IncRNA HOTAIR promotes cisplatin resistance in gastric cancer by targeting miR-126 to activate the PI3K/AKT/MRP1 genes. Tumor Biol: Tumour Biol: Nov 30, 2016 (Epub ahead of print).

76. Wang F, Li T, Zhang B, Li H, Wu Q, Yang L, Nie Y, Wu K, Shi Y and Fan D: MicroRNA-19a/b regulates multidrug resistance in human gastric cancer cells by targeting PTEN. Biochem Biophys Res Commun 434: 688-694, 2013.

77. Cao W, Yang W, Fan R, Li H, Jiang J, Geng M, Jin Y and Wu Y: miR-34a regulates cisplatin-induce gastric cancer cell death by modulating PI3K/AKT/survivin pathway. Tumor Biol 35: 1287-1295, 2014.

78. Eto K, Iwatsuki M, Watanabe M, Ida S, Ishimoto T, Iwagami S, Baba Y, Sakamoto Y, Miyamoto Y, Yoshida N and Baba H: The microRNA-21/PTEN pathway regulates the sensitivity of HER2-positive gastric cancer cells to trastuzumab. Ann Surg Oncol 21: 343-350, 2014.

79. Nishida N, Mimori K, Fabbri M, Yokobori T, Sudo T, Tanaka F, Shibata K, Ishii H, Doki Y and Mori M: MicroRNA-125a-5p is an independent prognostic factor in gastric cancer and inhibits the proliferation of human gastric cancer cells in combination with trastuzumab. Clin Cancer Res 17: 2725-2733, 2011.

80. Chou J, Shahi P and Werb Z: microRNA-mediated regulation of the tumor microenvironment. Cell Cycle 12: 3262-3271, 2013.

81. Yang TS, Yang XH, Chen X, Wang XD, Hua J, Zhou DL, Zhou B and Song ZS: MicroRNA-106b in cancer-associated fibroblasts from gastric cancer promotes cell migration and invasion by targeting PTEN. FEBS Lett 588: 2162-2169, 2014.

82.Zucker S and Vacirca J: Role of matrix metalloproteinases (MMPs) in colorectal cancer. Cancer Metastasis Rev 23: 101-117, 2004

83. Liu G, Jiang C, Li D, Wang R and Wang W: miRNA-34a inhibits EGFR-signaling-dependent MMP7 activation in gastric cancer. Tumor Biol 35: 9801-9806, 2014.

84. Tay Y, Rinn J and Pandolfi PP: The multilayered complexity of ceRNA crosstalk and competition. Nature 505: 344-352, 2014.

85. Balakirev ES and Ayala FJ: Pseudogenes: Are they 'junk' or functional DNA? Annu Rev Genet 37: 123-151, 2003.

86. Li L, Zhang J, Pan Q and Lei C: MicroRNA-23a regulates cell migration and invasion by target PTEN in gastric cancer. Int J Clin Exp Pathol 9: 877-887, 2016.

87. Yu B, Lv X, Su L, Li J, Yu Y, Gu Q, Yan M, Zhu Z and Liu B: miR-148a functions as a tumor suppressor by targeting CCK-BR via inactivating STAT3 and Akt in human gastric cancer. PLoS One 11: e0158961, 2016.

88. Tseng PC, Chen CL, Shan YS, Chang WT, Liu HS, Hong TM, Hsieh CY, Lin SH and Lin CF: An increase in integrin-linked kinase non-canonically confers NF- $\mathrm{BB}$-mediated growth advantages to gastric cancer cells by activating ERK1/2. Cell Commun Signal 12: 69, 2014.

89. Zhang L, Lei J, Fang ZL and Xiong JP: miR-128b is down-regulated in gastric cancer and negatively regulates tumour cell viability by targeting PDK1/Akt/NF- $\kappa \mathrm{B}$ axis. J Biosci 41: 77-85, 2016.

90. Sha M, Ye J, Zhang LX, Luan ZY, Chen YB and Huang JX: Celastrol induces apoptosis of gastric cancer cells by miR-21 inhibiting PI3K/Akt-NF- $\mathrm{KB}$ signaling pathway. Pharmacology 93: 39-46, 2014.

91. Paget S: The distribution of secondary growths in cancer of the breast. Lancet 133: 571-573 1889.
92. Lauschke VM, Mkrtchian S and Ingelman-Sundberg M: The role of microRNAs in liver injury at the crossroad between hepatic cell death and regeneration. Biochem Biophys Res Commun 482: 399-407, 2017.

93. Nagaraja AK, Creighton CJ, Yu Z, Zhu H, Gunaratne PH, Reid JG, Olokpa E, Itamochi H, Ueno NT, Hawkins SM, et al: A link between mir-100 and FRAP1/mTOR in clear cell ovarian cancer. Mol Endocrinol 24: 447-463, 2010.

94. Kwiatkowski DJ: Rhebbing up mTOR: New insights on TSC1 and TSC2, and the pathogenesis of tuberous sclerosis. Cancer Biol Ther 2: 471-476, 2003.

95. Kim LC, Cook RS and Chen J: mTORC1 and mTORC2 in cancer and the tumor microenvironment. Oncogene 36: 2191-2201, 2017.

96. Huang T, Kang W, Zhang B, Wu F, Dong Y, Tong JH, Yang W, Zhou Y, Zhang L, Cheng AS, et al: miR-508-3p concordantly silences NFKB1 and RELA to inactivate canonical NF- $\kappa B$ signaling in gastric carcinogenesis. Mol Cancer 15: 9, 2016.

97. Zhou X, Xia Y, Su J and Zhang G: Down-regulation of miR-141 induced by Helicobacter pylori promotes the invasion of gastric cancer by targeting STAT4. Cell Physiol Biochem 33: 1003-1012, 2014.

98. He B, Xiao YF, Tang B, Wu YY, Hu CJ, Xie R, Yang X, Yu ST, Dong $\mathrm{H}$, Zhao XY, et al: hTERT mediates gastric cancer metastasis partially through the indirect targeting of ITGB1 by microRNA-29a. Sci Rep 6: 21955, 2016.

99. Huang N, Wu Z, Lin L, Zhou M, Wang L, Ma H, Xia J, Bin J, Liao $Y$ and Liao W: miR-338-3p inhibits epithelial-mesenchymal transition in gastric cancer cells by targeting ZEB2 and MACC1/Met/Akt signaling. Oncotarget 6: 15222-15234, 2015.

100. Liu J, Wang X, Yang X, Liu Y, Shi Y, Ren J and Guleng B: miRNA423-5p regulates cell proliferation and invasion by targeting trefoil factor 1 in gastric cancer cells. Cancer Lett 347: 98-104, 2014.

101. Zhang J, Liu H, Hou L, Wang G, Zhang R, Huang Y, Chen X and Zhu J: Circular RNA_LARP4 inhibits cell proliferation and invasion of gastric cancer by sponging miR-424-5p and regulating LATS1 expression. Mol Cancer 16: 151, 2017.

102. Han X, Chen Y, Yao N, Liu H and Wang Z: MicroRNA let-7b suppresses human gastric cancer malignancy by targeting ING1. Cancer Gene Ther 22: 122-129, 2015.

103. Wang JY, Huang TJ, Chen FM, Hsieh MC, Lin SR, Hou MF and Hsieh JS: Mutation analysis of the putative tumor suppressor gene PTEN/MMAC1 in advanced gastric carcinomas. Virchows Arch 442: 437-443, 2003.

104. Velho S, Oliveira C, Ferreira A, Ferreira AC, Suriano G, Schwartz S Jr, Duval A, Carneiro F, Machado JC, Hamelin R and Seruca R: The prevalence of PIK3CA mutations in gastric and colon cancer. Eur J Cancer 41: 1649-1654, 2005.

105. Staal SP: Molecular cloning of the akt oncogene and its human homologues AKT1 and AKT2: Amplification of AKT1 in a primary human gastric adenocarcinoma. Proc Natl Acad Sci USA 84: 5034-5037, 1987.

106. Cheng Y, Li Y, Liu D, Zhang R and Zhang J: miR-137 effects on gastric carcinogenesis are mediated by targeting Cox-2-activated PI3K/AKT signaling pathway. FEBS Lett 588: 3274-3281, 2014.

107. Lu W, Xu Z, Zhang M and Zuo Y: miR-19a promotes epithelial-mesenchymal transition through PI3K/AKT pathway in gastric cancer. Int J Clin Exp Pathol 7: 7286-7296, 2014.

108. Li F, Liu B, Gao Y, Liu Y, Xu Y, Tong W and Zhang A: Upregulation of MicroRNA-107 induces proliferation in human gastric cancer cells by targeting the transcription factor FOXO1. FEBS Lett 588: 538-544, 2013.

109. Xu Y, Zhao F, Wang Z, Song Y, Luo Y, Zhang X, Jiang L, Sun Z, Miao Z and Xu H: MicroRNA-335 acts as a metastasis suppressor in gastric cancer by targeting Bcl-w and specificity protein 1. Oncogene 31: 1398-1407, 2011.

110. Zhao Y, Dong Q and Wang E: MicroRNA-320 inhibits invasion and induces apoptosis by targeting CRKL and inhibiting ERK and AKT signaling in gastric cancer cells. Onco Targets Ther 10: 1049-1058, 2017. 